\title{
Geometrostatic Manifolds of small ADM Mass
}

\author{
CHRISTINA SORMANI \\ CUNY Graduate Center and Lehman College \\ AND \\ IVA STAVROV ALLEN \\ Lewis \& Clark College
}

\begin{abstract}
We bound the locations of outermost minimal surfaces in geometrostatic manifolds whose ADM mass is small relative to the separation between the black holes and prove the Intrinsic Flat Stability of the Positive Mass Theorem in this setting. (c) 2000 Wiley Periodicals, Inc.
\end{abstract}

\section{Introduction}

Geometrostatic manifolds are asymptotically Euclidean solutions of the time symmetric vacuum Einstein-Maxwell constraint equations

$$
R(g)=2|E|^{2} \text { and } \operatorname{div} E=0 .
$$

They take the form of

$$
(M, g)=\left(\mathbb{R}^{3} \backslash P,(\chi \psi)^{2} \delta\right)
$$

where $P=\left\{p_{1}, \ldots p_{n}\right\}$ is the "set of holes", where $\delta$ is the Euclidean metric, and where $\psi>0, \chi>0$ on $\mathbb{R}^{3} \backslash P$ with $\Delta \chi=0, \Delta \psi=0$, and $\chi, \psi \rightarrow 1$ as $r \rightarrow \infty$ in $\mathbb{R}^{3}$. These manifolds were studied by Brill and Lindquist [BL63], Misner [Mis63] and Lichnerowicz [L44]. The conformal factors $\chi$ and $\psi$ are given by

$$
\chi(x)=1+\sum_{i=1}^{n} \frac{\alpha_{i}}{\rho_{i}} \quad \text { and } \quad \psi(x)=1+\sum_{i=1}^{n} \frac{\beta_{i}}{\rho_{i}}
$$

where $\alpha_{i}>0$ and $\beta_{i}>0$ are arbitrary and $\rho_{i}=\rho_{i}(x)=\left|p_{i}-x\right|$ is the Euclidean distance from $x$ to $p_{i}$. This metric on $M$ is complete and asympototically Euclidean as $x \rightarrow p_{i}$ and $|x| \rightarrow \infty$, so that we have $n+1$ ends. The electric field, $E$, is the gradient of the electrostatic potential, $\ln (\psi / \chi)$, up to a sign.

We see from [BL63] that the ADM mass of the $(n+1)^{s t}$ end, where $|x| \rightarrow \infty$, is

$$
m=m_{n+1}=\sum_{i=1}^{n}\left(\alpha_{i}+\beta_{i}\right),
$$


and that the $i^{t h}$ end, where $x \rightarrow p_{i}$, has ADM mass

$$
m_{i}=\alpha_{i}+\beta_{i}+\sum_{j \neq i} \frac{\left(\beta_{i} \alpha_{j}+\beta_{j} \alpha_{i}\right)}{r_{i, j}}
$$

and charge

$$
q_{i}=\beta_{i}-\alpha_{i}+\sum_{j \neq i} \frac{\left(\beta_{i} \alpha_{j}-\beta_{j} \alpha_{i}\right)}{r_{i, j}}
$$

with $r_{i, j}=\left|p_{i}-p_{j}\right|$ denoting the Euclidean distance from $p_{i}$ to $p_{j}$. We define the "separation factor" of the set of holes, $P$, to be

$$
\sigma=\sigma(P)=\min \left\{\sigma_{1}, \ldots, \sigma_{n},\left|p_{1}\right|, \ldots,\left|p_{n}\right|\right\} \text { where } \sigma_{i}=\min \left\{r_{i, j}: j \neq i\right\}
$$

When $q_{i}=0$ this manifold has zero scalar curvature and otherwise these manifolds have nonnegative scalar curvature.

Note that the Riemannian Schwarzschild black hole of mass $m_{1}$ is an example of a geometrostatic manifold with a single point $p_{1}=0$, charge, $q_{1}=0$, and mass, $m_{1}=2 \alpha_{1}=2 \beta_{1}$,

$$
\left(M, g_{\text {Sch }}\right)=\left(\mathbb{R}^{3} \backslash\left\{p_{1}\right\},(\chi \psi)^{2} \delta\right) \quad \text { where } \quad \chi(x)=\psi(x)=1+\frac{m_{1}}{2 \rho_{1}}
$$

and where $\delta$ is the Euclidean tensor. It has two asymptotically flat ends: as $r=$ $|x| \rightarrow \infty$ and as $r=|x| \rightarrow 0$. Between the two ends is a neck with a closed minimal surface $\Sigma=\left\{x:|x|=m_{1} / 2\right\}$ which is called an "apparent horizon". Note that

$$
\operatorname{Area}_{g}(\Sigma)=\left(1+\frac{m_{1}}{2\left(m_{1} / 2\right)}\right)^{4} 4 \pi\left(\frac{m_{1}}{2}\right)^{2}=16 \pi m_{1}^{2} \text { and } m=m_{n+1}=m_{1} .
$$

Any geometrostatic manifold, $(M, g)$, satisfying (1.2)-(1.3) may be viewed as a collection of $n$ black holes each with mass, $m_{i}$, and charge, $q_{i}$.

Recall the Positive Mass Theorem states that the ADM mass of an asymptotically Euclidean manifold with nonnegative scalar curvature is nonnegative, and when the ADM mass is 0 the manifold is Euclidean space [SY79]. This is easily seen to hold in the geometrostatic setting: by (1.4), we have

$$
m=\sum_{i=1}^{n}\left(\alpha_{i}+\beta_{i}\right) \geq 0 \text {. }
$$

and

$$
m=0 \quad \Longrightarrow \quad \alpha_{i}=\beta_{i}=0 \quad \Longrightarrow \quad(M, g)=\left(\mathbb{R}^{3}, \delta\right) .
$$

In particular there are no black holes if the ADM mass is zero.

The Penrose inequality states that if $M^{\prime}$ is an asymptotically Euclidean manifold of nonnegative scalar curvature whose boundary $\partial M^{\prime}$ is an outermost minimal surface then

$$
m_{A D M}\left(M^{\prime}, g\right) \geq \sqrt{\frac{\operatorname{Area}_{g}\left(\partial M^{\prime}\right)}{16 \pi}} .
$$


This was proven for $M^{\prime}$ with a connected boundary by Huisken-Ilmanen in [HI01]. Bray [Bra01] proved the inequality even when the boundary has more than one connected component.

Definition 1.1. In a Brill-Lindquist geometrostatic manifold, $M$, the outermost minimal surface $\Sigma_{i}$ about $p_{i}$ is a closed connected minimal surface, $\Sigma_{i}=\partial \Omega_{i} \subset \mathbb{R}^{3}$ where $p_{i} \in \Omega_{i}$, such that for any $\Omega \subset \mathbb{R}^{3}$ with $p_{i} \in \Omega$ and $\partial \Omega$ a closed minimal surface, we have $\Omega \subseteq \Omega_{i}$.

In the geometrostatic setting, each end $x \rightarrow p_{i}$ has such an outermost minimal surface, $\Sigma_{i}$ (see Example 2.3). Such minimal surfaces exist by the work of HuiskenIlmanen [HI01] which we review within Subsection 3.1. Note that it is possible when $p_{i}$ and $p_{j}$ are close enough, that $\Sigma_{i}=\Sigma_{j}$ (see Example 2.4). In fact such an example is computed numerically by Brill and Lindquist when $m_{i}$ and $m_{j}$ are large compared to $r_{i, j}$ [BL63]. In this paper we assume separation factor, $\sigma$, as in [1.7], is large compared to the ADM mass, $m$, of the manifold, $M$, and conclude that each outermost minimal surface, $\Sigma_{i}$, is distinct and is located in an annular region around $p_{i}$ :

Theorem 1.2. There exists a universal constant $C_{1} \gg 1$ such that if a geometrostatic manifold, $M$, has

$$
m<\frac{\sigma}{20 C_{1}},
$$

where $m$ is the ADM mass of the end at infinity as in (1.4) and $\sigma$ is the separation factor as in (1.7), then for all $i$ the $i^{\text {th }}$ outermost minimal surface of $M$ satisfies

$$
\Sigma_{i} \subseteq B_{\delta}\left(p_{i}, 2 C_{1} \sqrt{\operatorname{Area}_{g}\left(\Sigma_{i}\right) / \pi}\right) \backslash B_{\delta}\left(p_{i}, \frac{\alpha_{i} \beta_{i}}{4 C_{1}\left(\alpha_{i}+\beta_{i}\right)}\right) .
$$

In particular, since $2 C_{1} \sqrt{\operatorname{Area}_{g}\left(\Sigma_{i}\right) / \pi} \leq 8 C_{1} m<\sigma / 2$, the surfaces $\Sigma_{i}$ for distinct $i$ are disjoint.

This theorem is proven in Section 3 ,

Huisken-Ilmanen defined the exterior region, which we call an outermost region, in [HI01]. We review this notion carefully in Subsection 3.1 just stating the definition in our setting here:

Definition 1.3. The "outermost region", $M^{\prime} \subset M$, is

$$
M^{\prime}=\mathbb{R}^{3} \backslash \bigcup_{i=-n^{\prime}}^{n} \Omega_{i} .
$$

where each $\Omega_{i}$ is diffeomorphic to a ball and has outward minimizing boundary $\Sigma_{i}$. For $i \in\{1, \ldots, n\}$ these are the regions $\Omega_{i}$ containing $p_{i}$ that we defined above and for $i \leq 0$ these are possible additional outward minimizing regions (which we 
conjecture do not exist). Note that $M^{\prime}$ has one end as $|x| \rightarrow \infty$ and has an outermost minimizing boundary,

$$
\partial M^{\prime}=\Sigma=\bigcup_{i=-n^{\prime}}^{n} \Sigma_{i}
$$

and no closed interior minimal surfaces.

The outermost region satisfies the time symmetric vacuum Einstein-Maxwell equation. So it has nonnegative scalar curvature and, if the charge is 0 , then it has zero scalar curvature. By the Penrose Inequality in the form proven by Bray in [Bra01] we have

$$
m=m_{n+1}=m_{A D M}\left(M^{\prime}, g\right) \geq \sqrt{\frac{1}{16 \pi} \sum_{i=-n^{\prime}}^{n} \operatorname{Area}_{g}\left(\Sigma_{i}\right)} .
$$

We prove the following theorem in Section 3 ;

Theorem 1.4. Let $C_{1}$ be the constant of Theorem 1.2, and suppose that $M$ is geometrostatic with $\sigma>20 m C_{1}$. For all $1 \leq i \leq n$ there exists lengths $\gamma_{i} \leq 8 C_{1} m$ such that the outermost region $M^{\prime}$ of Definition 1.3 satisfies

$$
\mathbb{R}^{3} \backslash\left(\bigcup_{i=1}^{n} B_{\delta}\left(p_{i}, \gamma_{i}\right)\right) \subseteq M^{\prime} \subseteq \mathbb{R}^{3} \backslash\left(\bigcup_{i=1}^{n} B_{\delta}\left(p_{i}, \frac{\alpha_{i} \beta_{i}}{4 C_{1}\left(\alpha_{i}+\beta_{i}\right)}\right)\right)
$$

The reader may want to note that the definition and more detailed estimates involving $\gamma_{i}$ are presented in Sections 3 and 4, respectively.

It has been conjectured that if a sequence of pointed asymptotically flat manifolds, $\left(M_{k}^{\prime}, g_{k}, x_{k}\right)$, with nonnegative scalar curvature whose boundaries are outermost minimal surfaces, has $m_{A D M}\left(M_{k}^{\prime}\right) \rightarrow 0$, then $\left(M_{k}^{\prime}, g_{k}\right)$ converge in the pointed intrinsic flat sense to Euclidean space, $\left(\mathbb{R}^{3}, \delta, 0\right)$, assuming the manifolds are centered on well chosen points, $x_{k}$, which do not disappear down increasingly deep wells. When proposing this conjecture and proving it in the rotationally symmetric case, Lee and the second author demonstrated that this conjecture would be false if it were stated with a stronger notion of convergence [LS14]. Lan-Hsuan Huang, Dan Lee and the first author have proven this conjecture in the graph setting assuming additional hypotheses including one that requires all level sets to be outward minimizing [HLS16]. It is unknown whether the setting considered in [HLS16] can include multiple black holes. In Section 4, we prove this conjecture for geometrostatic manifolds:

Theorem 1.5. Let $\left(M_{k}, g_{k}\right)$ be a sequence of geometrostatic manifolds with outermost regions, $M_{k}^{\prime}$, such that

$$
m_{A D M}\left(M_{k}^{\prime}\right) \rightarrow 0 \quad \text { and } \quad \frac{m_{A D M}\left(M_{k}^{\prime}\right)}{\sigma\left(M_{k}\right)} \rightarrow 0
$$


where $\sigma\left(M_{k}\right)$ is the separation factor of the set of holes $P_{k}$ of $M_{k}$ as in (1.7). Assume furthermore that there is some $R_{0} \geq 0$ such that the set of accumulation points of $\rho\left(\cup_{k} P_{k}\right) \cap\left(R_{0}, \infty\right)$ is of measure 0 where $\rho(x)=|x|$.

Then $\left(M_{k}^{\prime}, g_{k}\right)$ converges in the pointed intrinsic flat sense to Euclidean space. More precisely, for almost all $R>R_{0}$ the ball $B_{g_{k}}(0, R) \subset\left(M_{k}^{\prime}, g_{k}\right)$ converges to the Euclidean ball $B_{\delta}(0, R) \subset \mathbb{E}^{3}$ in the intrinsic flat sense.

Before proving either theorem we present examples in Section 2 which illustrate why some aspects of the proofs are technically difficult. We review HuiskenIlmanen and prove Theorem 1.2 in Section 3. We review Intrinsic Flat Convergence particularly work of the first author with Lakzian [LS13], and prove Theorem 1.5 in Section 4. In the Appendix we provide additional information about geometrostatic manifolds needed within the paper.

The authors thank the organizers of the 2014 conference Geometric Analysis and Relativity Conference at the University of Science and Technology of China, at which this collaboration commenced. We further thank the organizers of the 2016 workshop Geometric Analysis and General Relativity at the Banff International Research Station, during which the final version of this work was formulated.

\section{Examples}

In this section we describe the location of the outermost region and outermost minimal surfaces in a variety of Brill-Lindquist geometrostatic manifolds.

Example 2.1. The Riemannian Schwarzschild manifold as in (1.8) can be depicted as in Figure 2.1 to emphasize that it has two asymptotically flat ends: one as $|x| \rightarrow 0$ and one as $|x| \rightarrow \infty$. The ends are not quite as flat as depicted here. The outermost minimal surface, $\Sigma=\left\{x:|x|=m_{1} / 2\right\}$, lies in the neck between the two ends and the outermost region, $M^{\prime}=\left\{x:|x|>m_{1} / 2\right\}$, lies above $\Sigma$ in this image.

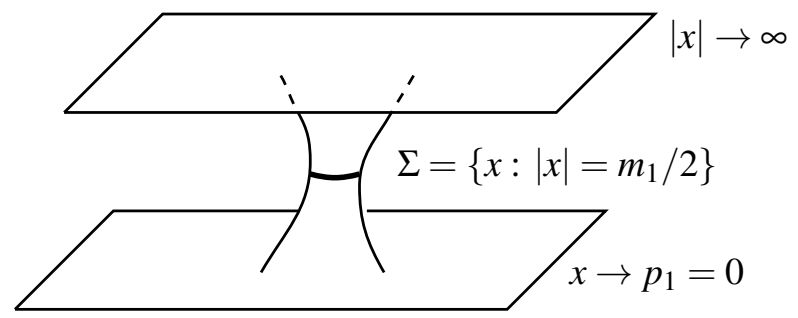

FIgURE 2.1. Example 2.1; The Riemannian Schwarzschild Manifold

Example 2.2. The extreme Reissner-Nordstrom black hole has a metric of the form (1.2)-1.3 with $n=1, \alpha_{1}>0$ and $\beta_{1}=0$. See Figure 2.2. It has one end as $|x| \rightarrow \infty$ which is asymptotically flat and one end as $x \rightarrow p_{1}$ which is asymptotically cylindrical. It is not a Brill-Lindquist geometrostatic manifold and has no outermost minimal surface $\Sigma$. One may view this example as having an infinitely long neck. 


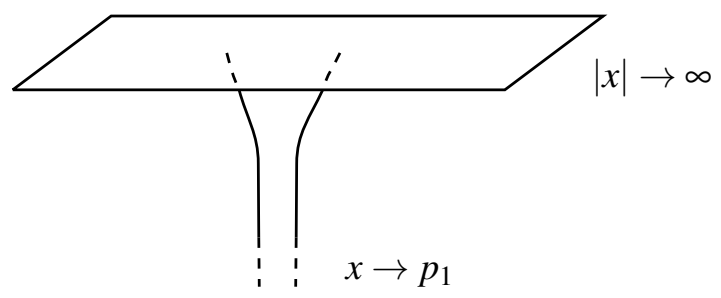

FIgURE 2.2. Example 2.2 Extreme Reissner-Nordstrom.

Example 2.3. A typical Brill-Lindquist geometrostatic manifold, $M$, satisfying the hypothesis of Theorems 1.2 and 1.5 is depicted in Figure 2.3. Here we have three black holes with small masses. The $p_{i}$ are located sufficiently far apart that they have distinct outermost minimal surfaces, $\Sigma_{i}$. The outermost region, $M^{\prime}$, lies above $\Sigma=\bigcup_{i} \Sigma_{i}$ in this image. If $\beta_{i}<<\alpha_{i}$ then the necks can be quite long as depicted here. Even with long necks, Theorem 1.5 implies that $M^{\prime}$ is close in the intrinsic flat sense to $\mathbb{E}^{3}$. The intrinsic flat distance essentially measures a volume between Euclidean space and $M^{\prime}$, and we prove these necks have small volume.

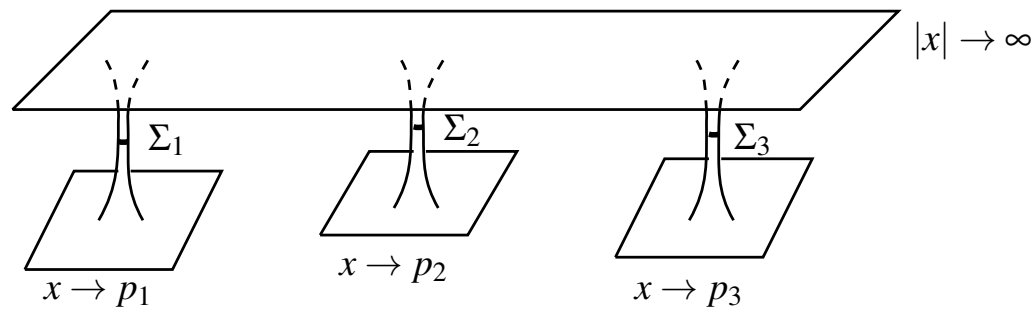

FIgURE 2.3. Example 2.3, $M$ as in Theorems 1.2 and 1.5.

Example 2.4. Brill and Lindquist demonstrated numerically that if the masses of two black holes are sufficiently large relative to the distance between them, then they share a single outermost minimal surface, $\Sigma_{1}=\Sigma_{2}$. Such a manifold, $M$, which fails to satisfy the hypothesis of Theorems 1.2 and 1.5, is depicted in Figure 2.4. The outermost region, $M^{\prime}$, lies above the $\Sigma_{i}$ in this image.

Example 2.5. In Figure 2.5 we see a sequence of outermost regions, $M_{k}^{\prime}$, satisfying the hypothesis of Theorem 1.5. In particular, $m_{A D M}\left(M_{k}^{\prime}\right) \rightarrow 0$. Here $n=1, \alpha_{1}>0$ and $\alpha_{1}>>\beta_{1}>0$ for each $M_{k}$, and so we have thinner and thinner necks which can be quite long. In the limit, the neck shrinks to a line segment which has no volume at all and thus disappears under intrinsic flat convergence. 


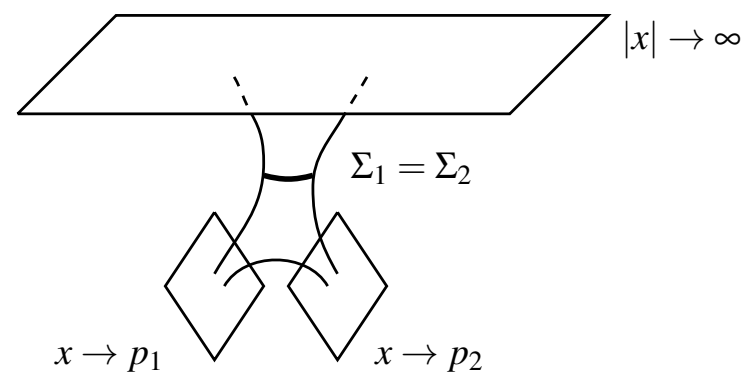

FIGURE 2.4. Example 2.4 Failing the hypothesis of Theorem 1.2 and 1.5

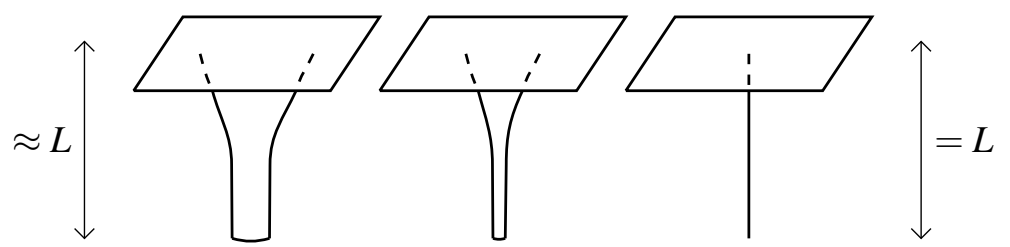

FIGURE 2.5. Example 2.5. A sequence of outermost regions as in Theorem 1.5

\section{Minimal Surfaces}

In this section we locate the outermost minimal surfaces, $\Sigma_{i}$, proving Theorem 1.2. In Subsection 3.1 we review Huisken-Ilmanen which proves the existence of the outermost minimal surfaces. In Subsection 3.3 we prove that an appropriately rescaled annular region within a geometrostatic manifold has bounded curvature and injectivity radius. These bounds are applied in Subsection 3.4 to provide a lower bound on $\operatorname{Area}_{g}\left(\Sigma_{i}\right)$. In Subsection 3.5 we prove that for all $\Sigma_{i}$ there is some $p \in P$ such that $\Sigma_{i} \cap \bar{B}_{\delta}\left(p, \sqrt{\operatorname{Area}_{g}\left(\Sigma_{i}\right) / \pi}\right) \neq \emptyset$. In Subsection 3.6 we combine these results and the Penrose Inequality to prove that if the separation factor $\sigma$ is large compared to the ADM mass $m$, then $\Sigma_{i} \subseteq B_{\delta}\left(p, 2 C_{1} \sqrt{\operatorname{Area}_{g}\left(\Sigma_{i}\right) / \pi}\right)$ for some $p \in P$. In Subsection 3.7 we apply the inversion proven in the Appendix to flip $p \in P$ to $\infty$ to prove that $\Sigma_{i}$ is not too close to any $p \in P$, thus completing the proof of Theorem 1.2 .

\subsection{A Review of Huisken-Ilmanen}

in [HI01], Huisken and Ilmanen provide a rigorous definition of an outermost minimal surface and prove its regularity. We review this here.

Let $M$ be a complete 3-manifold with asymptotically flat ends. Let $K_{1}$ be the closure of the union of the images of all smooth, compact, immersed minimal surfaces in $\mathrm{M}$. They observe that since the region near infinity is foliated by spheres of positive mean curvature, $K_{1}$ is compact. The trapped region $K$ is defined to be 
the union of $K_{1}$ together with the bounded components of $M \backslash K_{1}$. The set $K$ is clearly compact as well.

Let $M^{\prime}$ be any connected component of $M \backslash K$. This is considered to be an "exterior region". It has one asymptotically flat end and a compact boundary. In our paper we are considering specifically $M^{\prime}$ corresponding to the end as $r \rightarrow \infty$.

In Lemma 4.1 of [HI01], Huisken and Ilmanen prove that $M^{\prime}$ is connected and asymptotically flat, has a compact, minimal boundary, and contains no other compact minimal surfaces (even immersed). In addition $M^{\prime}$ is diffeomorphic to $\mathbb{R}^{3}$ minus a finite number of regions diffeomorphic to open 3-balls with disjoint closures. The boundary of $M^{\prime}$ minimizes area in its homology class. This $M^{\prime}$ is the outermost region we have defined in our introduction.

Let $\Sigma$ be any connected component of $\partial M^{\prime}$. Huisken-Ilmanen proved that

$$
m_{A D M}\left(M^{\prime}, g\right) \geq \sqrt{\frac{\text { Area }_{g}(\Sigma)}{16 \pi}}
$$

which implies the Penrose Inequality if $\partial M^{\prime}$ were connected [HI01]. Bray [Bra01] proved the Penrose Inequality as in (1.12) even when the boundary has more than one connected component.

Applying this to our paper, we have an outermost or exterior region

$$
M^{\prime}=\mathbb{R}^{3} \backslash \bigcup_{\alpha} U_{\alpha}
$$

where $U_{\alpha}$ are diffeomorphic to three dimensional balls with stable minimal boundaries, $\Sigma_{\alpha}=\partial U_{\alpha}$. Note that every $p_{i}$ must lie in one of the $U_{\alpha}$ and that if $p_{i} \in U_{\alpha}$ then $U_{\alpha}=\Omega_{\alpha}$ of Definition 1.1. Recall that it is possible that some $\Omega_{i}=\Omega_{j}$. It is possible that there are some additional $U_{\alpha}$ which do not contain any $p_{i}$ for $i=1$ to $n$. We set these $U_{\alpha}=\Omega_{i}$ with $i \leq 0$ so that we may simply write:

$$
M^{\prime}=\mathbb{R}^{3} \backslash \bigcup_{i=-n^{\prime}}^{n} \Omega_{i} \text { and } \partial M^{\prime}=\bigcup_{i=-n^{\prime}}^{n} \Sigma_{i} \text { where } \Sigma_{i}=\partial \Omega_{i} .
$$

Observe that we have

$$
\Omega_{i} \neq \Omega_{j} \Longrightarrow \Omega_{i} \cap \Omega_{j}=\emptyset .
$$

Conjecture 3.1. We conjecture that for every $\Omega_{\alpha}$ there is a $p_{i} \in \Omega_{\alpha}$ so that $M^{\prime}=$ $\mathbb{R}^{3} \backslash \bigcup_{i=1}^{n} \Omega_{i}$.

\subsection{Minimal surfaces in a Conformally Flat Manifold}

Suppose

$$
(M, g)=\left(\mathbb{R}^{3} \backslash P, \Psi^{2} \delta\right)
$$

where $P=\left\{p_{1}, \ldots p_{n}\right\}, \delta$ is the Euclidean metric, and $\Psi>0$ on $\mathbb{R}^{3} \backslash P$. We will not require $\Psi^{2}=(\chi \psi)^{2}$ in the beginning of this subsection. 
If $\Sigma$ is a closed surface in this manifold the area of $\Sigma$ with respect to the metric $g$ is

$$
\operatorname{Area}_{g}(\Sigma)=\int_{x \in \Sigma} d \sigma_{g}=\int_{x \in \Sigma} \Psi^{2}(x) d \sigma_{\delta}
$$

If we vary $\Sigma_{t}$ with respect to an arbitrary variational field $\mathbf{v}=f \mathbf{n}$ where $\mathbf{n}$ is the outward normal, we see that

$$
\left.\frac{d}{d t} \operatorname{Area}_{g}\left(\Sigma_{t}\right)\right|_{t=0}=\int_{x \in \Sigma}\left(2 \Psi(x) \nabla \Psi_{x} \cdot \mathbf{n}+\Psi^{2}(x) H_{x}\right) f(x) d \sigma_{\delta}
$$

where $H_{x}$ is the outward pointing mean curvature of $\Sigma$ as a submanifold of $(M, \delta)$ and $\nabla=\nabla_{\delta}$ is the gradient with respect to the Euclidean metric $\delta$. Thus if $\Sigma_{t}$ is a minimal surface in $(M, g)$ then

$$
2 \Psi(x) \nabla \Psi_{x} \cdot \mathbf{n}+\Psi^{2}(x) H_{x}=0 \quad \forall x \in \Sigma
$$

and

$$
H_{x}=-2 \frac{\nabla \Psi_{x} \cdot \mathbf{n}}{\Psi(x)} \quad \forall x \in \Sigma .
$$

On any surface, the mean curvature is the sum of the principal curvatures, $H=$ $\lambda_{1}+\lambda_{2}$, and while the Gauss curvature $K=\lambda_{1} \lambda_{2}$ and so $H^{2}-4 K \geq 0$. By GaussBonnet, the Euler charateristic satisfies

$$
2 \pi \chi(\Sigma)=\int_{\Sigma} K d \sigma_{\delta} \leq \frac{1}{4} \int_{\Sigma} H^{2} d \sigma_{\delta} .
$$

Combining this with (3.9) we see that any minimal surface satisfies:

$$
2 \pi \chi(\Sigma) \leq \frac{1}{4} \int_{\Sigma}\left(-2 \frac{\nabla \Psi_{x} \cdot \mathbf{n}}{\Psi(x)}\right)^{2} d \sigma_{\delta}
$$

which also implies that

$$
\begin{aligned}
2 \pi \chi(\Sigma) & \leq \int_{\Sigma} \frac{\left(\nabla \Psi_{x} \cdot \mathbf{n}\right)^{2}}{\Psi^{2}(x)} d \sigma_{\delta} \\
& \leq \int_{\Sigma} \frac{\left|\nabla \Psi_{x}\right|^{2}}{\Psi^{4}(x)} \Psi^{2}(x) d \sigma_{\delta} \\
& \leq \max _{x \in \Sigma}\left\{\frac{\left|\nabla \Psi_{x}\right|^{2}}{\Psi^{4}(x)}\right\} \int_{\Sigma} \Psi^{2}(x) d \sigma_{\delta} \\
& =\max _{x \in \Sigma}\left\{\frac{\left|\nabla \Psi_{x}\right|^{2}}{\Psi^{4}(x)}\right\} \operatorname{Area}_{g}(\Sigma) .
\end{aligned}
$$

Note that we have equality iff $\frac{\left|\nabla \Psi_{x}\right|^{2}}{\Psi^{4}(x)}$ is constant on $\Sigma,(\nabla \Psi \cdot \mathbf{n})=|\nabla \Psi|$ and $H^{2}=4 K$ where $H$ and $K$ are the mean and Gauss curvatures of the surface with respect to the Euclidean metric on $M$. Note further that $(\nabla \Psi \cdot \mathbf{n})=|\nabla \Psi|$ iff $\nabla \Psi$ is perpendicular to the surface iff $\Psi$ is constant on the surface. This implies the following proposition: 
Proposition 3.2. The area of a minimal surface in $(M, g)$ as in (3.5) is bounded below by:

$$
\operatorname{Area}_{g}(\Sigma) \geq 2 \pi \chi(\Sigma)\left(\max _{x \in \Sigma}\left\{\frac{\left|\nabla \Psi_{x}\right|^{2}}{\Psi^{4}(x)}\right\}\right)^{-1}
$$

and we have equality iff $\Psi$ and $\left|\nabla \Psi_{x}\right|$ are constant on $\Sigma$ and $H^{2}=4 K$ where $H$ and $K$ are the mean and Gauss curvatures of the surface with respect to the Euclidean metric on $M$.

In particular when $g$ is a metric with positive scalar curvature, we know by Huisken-Ilmanen that any outward minimizing surface is smooth minimal surface diffeomorphic to a sphere. So we have (3.16) with $\chi(\Sigma)=2$. Furthermore, by the Penrose Inequality, as proven in Huisken-Ilmanen, we have

$$
\operatorname{Area}_{g}(\Sigma) \leq 16 \pi m^{2} \text { where } m=m_{A D M}(M)
$$

with equality iff $M$ is isometric to Schwarzschild space. Combining this with the previous proposition we have the following:

Proposition 3.3. If $(M, g)$ as in (3.5) has positive scalar curvature and $\Sigma$ is outward minimizing then

$$
16 \pi m^{2} \geq 4 \pi\left(\max _{x \in \Sigma}\left\{\frac{\left|\nabla \Psi_{x}\right|^{2}}{\Psi^{4}(x)}\right\}\right)^{-1}
$$

and we have equality iff $\Psi$ and $\left|\nabla \Psi_{x}\right|$ are constant on $\Sigma$ and $H^{2}=4 K$ and $(M, g)$ is isometric to Schwarzschild space.

This immediately implies the following:

Proposition 3.4. Let $(M, g)$ be a geometrostatic manifold with $\Psi(x)=\psi(x) \chi(x)$ satisfying (1.3). Then by (3.9) any minimal surface $\Sigma$ satisfies:

$$
H_{x}=-2\left(\frac{\nabla \psi_{x} \cdot \mathbf{n}}{\psi(x)}+\frac{\nabla \chi_{x} \cdot \mathbf{n}}{\chi(x)}\right) \quad \forall x \in \Sigma .
$$

If in addition the minimal surface is outward minimizing, we have

$$
\begin{aligned}
4 \pi & \leq \int_{\Sigma}\left(\frac{\nabla \psi_{x} \cdot \mathbf{n}}{\psi(x)}+\frac{\nabla \chi_{x} \cdot \mathbf{n}}{\chi(x)}\right)^{2} d \sigma_{\delta} \\
& \leq \operatorname{Area}_{g}(\Sigma) \max _{x \in \Sigma}\left(\frac{\nabla \psi_{x} \cdot \mathbf{n}}{\psi^{2}(x) \chi(x)}+\frac{\nabla \chi_{x} \cdot \mathbf{n}}{\chi^{2}(x) \psi(x)}\right)^{2} \\
& \leq 16 \pi m^{2} \max _{x \in \Sigma}\left(\frac{\left|\nabla \psi_{x}\right|}{\psi^{2}(x) \chi(x)}+\frac{\left|\nabla \chi_{x}\right|}{\chi^{2}(x) \psi(x)}\right)^{2}
\end{aligned}
$$

and we have equality iff $\psi(x) \chi(x)$ and $\nabla(\psi(x) \chi(x))$ are constant on $\Sigma$ and $H^{2}=4 K$ and $(M, g)$ is isometric to Schwarzschild space. 
Example 3.5. We can apply this proposition to Schwarzschild space $M_{\text {Sch }}$, satisfying (1.8) with mass $m=\alpha_{1}+\beta_{1}=2 \alpha_{1}$ to verify that the level set $\rho_{1}^{-1}(m / 2)$ is a minimal surface and prove it is the only outermost minimizing surface in $M_{\text {Sch. }}$. On $\rho_{1}^{-1}(m / 2)$ we have

$$
\chi(x)=\psi(x)=1+\beta_{1} / \rho_{1}(x)=1+(m / 2) /(m / 2)=1+1=2
$$

and

$$
\nabla \chi_{x}=\nabla \psi_{x}=\left(-\beta_{1} / \rho_{1}^{2}(x)\right) \nabla \rho_{1}=-(m / 2) /(m / 2)^{2} \nabla \rho_{1}=-(2 / m) \nabla \rho_{1}
$$

Since $H_{x}=2 / \rho_{1}=2 /(m / 2)=4 / m$ and

$$
-2\left(\frac{\nabla \psi_{x} \cdot \mathbf{n}}{\psi(x)}+\frac{\nabla \chi_{x} \cdot \mathbf{n}}{\chi(x)}\right)=-2(-2 / m)(1 / 2)-2(-2 / m)(1 / 2)=4 / m,
$$

we have (3.19) and $\rho_{1}^{-1}(m / 2)$ is a minimal surface in $(M, g)$. So now we know our outermost region $M^{\prime} \subset \rho_{1}^{-1}[m / 2, \infty)$.

Next suppose $\Sigma \subset M^{\prime}$ were an outermost minimizing surface, then by Proposition 3.4 we have

$$
\begin{aligned}
4 \pi & \leq 16 \pi m^{2} \max _{x \in \Sigma}\left(\frac{\left|\nabla \psi_{x}\right|}{\psi^{2}(x) \chi(x)}+\frac{\left|\nabla \chi_{x}\right|}{\chi^{2}(x) \psi(x)}\right)^{2} \\
& =16 \pi m^{2} \max _{x \in \Sigma}\left(\frac{2\left|\nabla \psi_{x}\right|}{\psi^{3}(x)}\right)^{2} \\
& =16 \pi m^{2} \max _{x \in \Sigma} \frac{4\left|-(m / 2) / \rho_{1}^{2}(x)\right|^{2}\left|\nabla \rho_{1}\right|^{2}}{\left|1+(m / 2) / \rho_{1}(x)\right|^{6}} \\
& =16 \pi m^{2} \max _{x \in \Sigma} \frac{\left(m \rho_{1}(x)\right)^{2}}{\left|\rho_{1}(x)+(m / 2)\right|^{6}} \quad \text { because }\left|\nabla \rho_{1}\right|=1 \\
& =16 \pi m^{2} \max _{x \in \Sigma} F^{2}\left(\rho_{1}(x), m / 2\right) \text { where } F(\rho, \beta)=2 \rho \beta /(\rho+\beta)^{3} .
\end{aligned}
$$

For fixed $\beta=\beta_{1}=m / 2, F(\rho, \beta)$ converges to 0 as $\rho \rightarrow 0$ and as $\rho \rightarrow \infty$. F increases to a single critical point at $\rho=\beta / 2=m / 4$ and then decreases. Since $\Sigma \subset M^{\prime}$ implies $\rho_{1}(x) \geq \beta_{1}=m / 2$, the maximum of $F$ occurs at $\rho=m / 2$. Thus we have

$$
\begin{aligned}
4 \pi & \leq 16 \pi m^{2} \max _{\rho \in[m / 2, \infty)} F^{2}(\rho, m / 2) \leq 16 \pi m^{2} F^{2}(m / 2, m / 2) \\
& =16 \pi m^{2}\left(\frac{2(m / 2)(m / 2)}{((m / 2)+(m / 2))^{3}}\right)^{2}=16 \pi m^{2}\left(\frac{m^{2} / 2}{m^{3}}\right)^{2}=4 \pi .
\end{aligned}
$$

Thus we have equality in Proposition 3.4 , which implies that

$$
\frac{m^{2} \rho_{1}^{2}(x)}{\left|\rho_{1}(x)+(m / 2)\right|^{6}}=\frac{m^{2}(m / 2)^{2}}{|(m / 2)+(m / 2)|^{6}} \text { for all } x \in \Sigma \text {. }
$$

So $\rho_{1}(x)=m / 2$ for all $x \in \Sigma$. Thus we have confirmed that $\rho_{1}^{-1}(m / 2)$ is the only outermost minimizing surface in $M_{\text {Sch. }}$. 
We conjecture more generally that if $(M, g)$ is a geometrostatic manifold as in (1.2), then the only outermost minimizing surfaces are the $\Sigma_{i}$ defined in 1.1

\subsection{Estimates on the curvature and the injectivity radius}

In this section we prove that an appropriately rescaled annular region within a geometrostatic manifold has bounded curvature and injectivity radius. These bounds will be applied later to locate the outermost minimal surfaces in these manifolds.

Fix a geometrostatic manifold $\left(\mathbb{R}^{3} \backslash P, g\right)$ and fix some $i$ with $1 \leq i \leq n$. We assume there is some positive length, $c>0$, such that

$$
\sigma_{i}=\min _{j \neq i}\left\{\left|p_{i}-p_{j}\right|\right\}>5 c \quad \text { or } \quad \sigma_{i}^{\text {out }}=\max _{j \neq i}\left\{\left|p_{i}-p_{j}\right|\right\}<\frac{1}{5} c .
$$

Consider a Euclidean annulus

$$
\mathscr{A}=\left\{u \in \mathbb{R}^{3}\left|\frac{1}{4} \leq\right| u \mid \leq 4\right\}
$$

and the mapping

$$
\Phi: \mathscr{A} \rightarrow \mathbb{R}^{3} \backslash P \text { by } u \mapsto p_{i}+c u .
$$

By our choice of $c$ in (3.34) we know that $\Phi(\mathscr{A})$ avoids $P$ by a definite amount. In fact $\Phi^{-1}\left(P \backslash\left\{p_{i}\right\}\right)$ either lies beyond the outer ring of the annulus when $\sigma_{i}<5 c$ or lies within the inner ring when $\sigma_{i}^{\text {out }}>\frac{1}{5} c$.

The scaled pullback metric $g_{c, \Phi}:=c^{-2} \Phi^{*} g$ on the annulus is easily seen to be

$$
\left(1+\frac{\alpha_{i} / c}{|u|}+\varphi_{\alpha}(u)\right)^{2}\left(1+\frac{\beta_{i} / c}{|u|}+\varphi_{\beta}(u)\right)^{2} \delta
$$

where

$$
\varphi_{\alpha}(u)=\sum_{j \neq i} \frac{\alpha_{j}}{\left|c u+p_{i}-p_{j}\right|} \text { and } \varphi_{\beta}(u)=\sum_{j \neq i} \frac{\beta_{j}}{\left|c u+p_{i}-p_{j}\right|} .
$$

Before we state the main results of this section, we introduce some terminology which will make our statements more efficient.

Definition 3.6. Let $\psi(u)$ be a function (or a tensor field) defined on the annulus $\mathscr{A}=\left\{u \in \mathbb{R}^{3}\left|\frac{1}{4} \leq\right| u \mid \leq 4\right\}$. We say that $\psi$ is controllable by $K_{0}$ whenever there exists a positive increasing function $P$, independent of $\psi$, such that

$$
\|\psi\|_{C^{0}(\mathscr{A})} \leq P\left(K_{0}\right)
$$

Furthermore, we say that $\psi$ is controllable by $K_{0}$ with all of its derivatives whenever all of the Euclidean partial derivatives $\partial_{u}^{l}$ (of the components of $\psi(u)$ ) are controllable by $K_{0}$.

Here are the two main results of this subsection. 
Proposition 3.7. Assume there is a positive length, $c>0$, that satisfies (3.34) and consider the scaled pullback metric $g_{c, \Phi}$ on $\mathscr{A}$ defined in (3.36)-3.37). Observe that

(1) $g_{c, \Phi} \geq \delta$.

(2) There exist constants $k_{1}$ and $k_{2}$ such that $k_{1} \delta \leq g_{c, \Phi} \leq k_{2} \delta$ and such that both $\frac{k_{1}}{k_{2}}$ and $\frac{k_{2}}{k_{1}}$ are controllable by $m /\left(\sigma_{i}+c\right)$.

(3) The Christoffel symbols of $g_{c, \Phi}$ are controllable by $m /\left(\sigma_{i}+c\right)$ with all of their derivatives.

(4) The (sectional) curvature of $g_{c, \Phi}$ is controllable by $m /\left(\sigma_{i}+c\right)$ with all of its derivatives.

We also control the injectivity radius. Note that Cheeger-Gromov-Taylor estimate the injectivity radius in a far more general setting in [CGT82].

Proposition 3.8. Assume there is a positive length, $c>0$, that satisfies (3.34) and let $m /\left(\sigma_{i}+c\right) \leq 1$. Consider the scaled pullback metric $g_{c, \Phi}$ defined in (3.36)(3.37). There is a uniform lower bound, $i_{0}>0$, on the injectivity radii of $g_{c, \Phi}$ over $\mathscr{A}^{\prime}=\left\{u \in \mathbb{R}^{3}\left|\frac{1}{2} \leq\right| u \mid \leq 2\right\}$.

Remark 3.9. Note that the explicit bound, $m /\left(\sigma_{i}+c\right) \leq 1$, given in Proposition 3.8 is not optimal, and has only been chosen for simplicity.

Before proving these propositions, we first prove a series of general lemmas.

Lemma 3.10. Let $v \in \mathbb{R}$. The l-th order partial derivatives of $\xi \mapsto|\xi|^{v}$ on $\mathbb{R}^{3}$ satisfy point-wise estimate

$$
\left|\partial^{l}\left(|\xi|^{v}\right)\right| \leq C_{l, v}|\xi|^{v-l}
$$

where the constant $C_{l, v}$ depends only on $l$ and $v$.

Proof. We first prove the lemma in the case of $v=1$ :

$$
\left|\partial^{l}(|\xi|)\right| \leq C(l) \cdot|\xi|^{1-l},
$$

where the constant $C(l)$ depends only on $l$. We do so by induction on $l$. A direct computation verifies the base cases $l \leq 2$. For $l \geq 3$ the claim follows from the inductive hypothesis and

$$
\partial^{l}\left(|\xi|^{2}\right)=2|\xi| \partial^{l}(|\xi|)+\sum_{i=1}^{i=l-1}\left(\begin{array}{l}
l \\
i
\end{array}\right) \partial^{i}(|\xi|) \partial^{l-i}(|\xi|) .
$$

For general values of $v$ observe that the derivative $\partial^{l}\left(|\xi|^{v}\right)$ is a linear combination of terms of the form

$$
|\xi|^{v-k} \partial^{l_{1}}(|\xi|) \ldots \partial^{l_{k}}(|\xi|), \quad 1 \leq k \leq l
$$

with coefficients which depend only on $v$ and positive integers $l_{1}, \ldots, l_{k}$ which satisfy $l_{1}+\ldots+l_{k}=l$. The claim of our lemma is now a consequence of (3.41). 
Lemma 3.11. Let $\varphi \geq 0$ be a smooth function defined on the annulus, $\mathscr{A}$, defined in (3.35) Let a be a positive real number and let

$$
f(u)=\ln \left(1+\frac{a}{|u|}+\varphi(u)\right) .
$$

For each integer value of $l \geq 0$ there exist polynomials $P_{l}$ whose (positive) coefficients are independent of $a$ and $\varphi$ such that

$$
\|d f\|_{C^{l}(\mathscr{A})} \leq P_{l}\left(\|\varphi\|_{C^{l+1}(\mathscr{A})}\right) .
$$

Proof. Consider the function $\tilde{f}=e^{f}$. A straightforward induction argument shows that the components of the $l$-th derivatives of $d f$ are polynomials in $\tilde{f}^{-1} \partial^{i} \tilde{f}$ whose coefficients depend only on $l$. Thus, it suffices to prove point-wise bounds on $\tilde{f}^{-1} \partial^{i} \tilde{f}$ in terms of $\|\varphi\|_{C^{i}(\mathscr{A})}$ and constants which depend only on $i$.

By virtue of the fact that the annulus $\mathscr{A}$ is compact and bounded away from the origin we know that there is a constant $c_{i}$ depending only on $i$ such that

$$
\left|\partial^{i} \tilde{f}\right| \leq 1+c_{i} a+\|\varphi\|_{C^{i}(\mathscr{A})} \text {. }
$$

Thus,

$$
\left|\frac{\partial^{i} \tilde{f}}{\tilde{f}}\right| \leq \frac{1+c_{i} a}{1+a / 4}+\|\varphi\|_{C^{i}(\mathscr{A})} \leq 4\left(1+c_{i}\right)+\|\varphi\|_{C^{i}(\mathscr{A})} .
$$

This completes our proof.

Lemma 3.12. Assume there is a positive length, $c>0$, that satisfies (3.34) and consider the scaled pullback metric $g_{c, \Phi}$ on $\mathscr{A}$ defined in (3.36)-(3.37). Then for every integer $l \geq 0$ there exists a constant $C_{l}$ which depends only on $l$ such that

$$
\left\|\varphi_{\alpha}\right\|_{C^{l}(\mathscr{A})},\left\|\varphi_{\beta}\right\|_{C^{l}(\mathscr{A})} \leq C_{l} \frac{m}{\sigma_{i}+c} .
$$

Proof. As a consequence of Lemma 3.10 we have that

$$
\left|\partial_{u}^{l}\left(\frac{\alpha_{j}}{\left|c u+p_{i}-p_{j}\right|}\right)\right| \leq C_{l}^{\prime} \frac{\alpha_{j} c^{l}}{\left|c u+p_{i}-p_{j}\right|^{l+1}},
$$

where the constant $C_{l}^{\prime}$ depends only on $l$.

There are two cases in our hypothesis that $c$ satisfies (3.34). In the case where $\sigma_{i}>5 c$, we have $\left|p_{i}-p_{j}\right|>5 c$ for all $j \neq i$, and so we have

(3.50) $\left|c u+p_{i}-p_{j}\right| \geq\left|p_{i}-p_{j}\right|-c|u| \geq \sigma_{i}-4 c>\sigma_{i}-\frac{4 \sigma_{i}}{5}=\frac{\sigma_{i}}{5}>\frac{\sigma_{i}+c}{10}>\frac{\sigma_{i}+c}{40}$.

In the case where $\sigma_{i}^{\text {out }}<\frac{1}{5} c$ we have $\left|p_{i}-p_{j}\right|<c / 5$ and so we have

$$
\left|c u+p_{i}-p_{j}\right| \geq c|u|-\left|p_{i}-p_{j}\right| \geq \frac{c}{4}-\frac{c}{5}=\frac{c}{20}>\frac{\sigma_{i}+c}{40}
$$

because $\sigma_{i}<\sigma_{i}^{\text {out }}$.

In both cases, it follows from (3.49) and $\sum_{j} \alpha_{j}<m$ that

$$
\left|\partial_{u}^{l} \varphi_{\alpha}(u)\right| \leq C_{l}^{\prime} \sum_{j \neq i} \alpha_{j} \frac{c^{l}}{\left(\frac{c+\sigma_{i}}{40}\right)^{l+1}}<C_{l}^{\prime}\left(\frac{m}{\sigma_{i}+c}\right)\left(\frac{c}{\sigma_{i}+c}\right)^{l}<C_{l}^{\prime}\left(\frac{m}{\sigma_{i}+c}\right) .
$$

The same argument applies to $\varphi_{\beta}$. 
We now prove Proposition 3.7 .

Proof of Proposition 3.7 The first claim that $g_{c, \Phi} \geq \delta$ is immediate from 3.37.

By (3.37), the fact that $\varphi_{\alpha}>0$ and $\varphi_{\beta}>0$, the fact that $4>|u|>1 / 4$ on $\mathscr{A}$, and Lemma 3.12 with $l=0$ we have

$$
\left(1+\frac{\alpha_{i}}{4 c}\right)^{2}\left(1+\frac{\beta_{i}}{4 c}\right)^{2} \delta \leq g_{c, \Phi} \leq\left(1+4 \frac{\alpha_{i}}{c}+C_{0} \frac{m}{\sigma_{i}+c}\right)^{2}\left(1+4 \frac{\beta_{i}}{c}+C_{0} \frac{m}{\sigma_{i}+c}\right)^{2} \delta .
$$

Thus we have the second claim of Proposition 3.7

To prove the remaining claims we express $g_{c, \Phi}$ in the form of $e^{2 f} \delta$ where

$$
f(u)=\ln \left(1+\frac{\alpha_{i} / c}{|u|}+\varphi_{\alpha}(u)\right)+\ln \left(1+\frac{\beta_{i} / c}{|u|}+\varphi_{\beta}(u)\right)
$$

for functions $\varphi_{\alpha}$ and $\varphi_{\beta}$ of Lemma 3.12. In fact, by applying Lemmas 3.11 and 3.12 we see that there exist polynomials $P_{l}$ whose (positive) coefficients depend only on $l$ such that

$$
\|d f\|_{C^{l}(\mathscr{A})} \leq P_{l}\left(\frac{m}{\sigma_{i}+c}\right) .
$$

The claim about the Christoffel symbols of $g_{c, \Phi}$ is now immediate from the fact that $\Gamma_{i j}^{k}$ can be expressed in terms of components of $d f$. This also means that the components $R_{i j k}^{l}$ of the Riemann curvature tensor are bounded by a (universal) polynomial in $\frac{m}{\sigma_{i}+c}$. Since $g_{c, \Phi}^{-1} \leq \delta^{-1}$, the same applies to the sectional curvatures

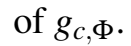

We now prove Proposition 3.8 .

Proof of Proposition 3.8 It follows from Proposition 3.7 that the Christoffel symbols of $g_{c, \Phi}$ are bounded over $\mathscr{A}$, together with all of their derivatives. Thus, the Cauchy-Picard Theorem implies the uniform time of existence $T$ for all geodesics $\gamma$ of $g_{c, \Phi}$ with

$$
\gamma(0) \in \mathscr{A}^{\prime}, \quad\left\|\gamma^{\prime}(0)\right\|_{\delta} \leq 1
$$

In particular, we know that for each $Q \in \mathscr{A}^{\prime}$ the mapping $\exp _{Q}$ is defined on

$$
B_{\delta}(0, T) \supseteq B_{g_{c, \Phi}}(0, T) .
$$

The fact that for each such $Q$ the mapping $\exp _{Q}$ is a local diffeomorphism follows from the Inverse Function Theorem. In fact, the proof of the Inverse Function Theorem shows that if

$$
\left\|\mathrm{Id}-\left.D\right|_{v}\left(\exp _{Q}\right)\right\|_{\delta}<\frac{1}{2}
$$

for all $v \in B_{\delta}\left(Q, 2 r_{*}\right)$ then $\exp _{Q}$ maps diffeomorphically onto the ball $B_{\delta}\left(Q, r_{*}\right)$.

We proceed by showing that a radius $r_{0}$ can be chosen independently of $Q$ so that the estimate (3.58) holds for all $v \in B_{g_{c, \Phi}}\left(Q, 2 r_{0}\right)$. Since

$$
B_{\delta}\left(Q, 2 r_{0} / k_{2}\right) \subseteq B_{g_{c, \Phi}}\left(Q, 2 r_{0}\right) \text { and } B_{g_{c, \Phi}}\left(Q, k_{1} r_{0} / k_{2}\right) \subseteq B_{\delta}\left(Q, r_{0} / k_{2}\right)
$$


for the constants $k_{1}$ and $k_{2}$ addressed in Proposition 3.7, the estimate (3.58) and the proof of the Implicit Function Theorem imply that $\exp _{Q}$ is a diffeomorphism on $B_{g_{c, \Phi}}\left(Q, k_{1} r_{0} / k_{2}\right)$. This observation makes the claim of our proposition a consequence of the fact that (3.58) holds for all $v \in B_{g_{c, \Phi}}\left(Q, 2 r_{0}\right)$.

As $\left.D\right|_{v}\left(\exp _{Q}\right)$ is identity on the span of $v$, it suffices to study $\left.D\right|_{v}\left(\exp _{Q}\right)$ on the orthogonal complement of $v$. There the mapping is given by the Jacobi vector fields $Y$ along the $g_{c, \Phi}$-unit speed geodesic $\gamma(t)=\exp _{Q}\left(t \cdot \frac{1}{\|v\|} v\right)$ :

$$
\left.D\right|_{v}\left(\exp _{Q}\right): w \mapsto \frac{1}{\|v\|} Y(\|v\|), Y(0)=0, Y^{\prime}(0)=w .
$$

Note that here $\|v\|$ is taken with respect to $g_{c, \Phi}$. For the purposes of addressing (3.58) it suffices to work with $w$ which are unit with respect to $\delta$. Note that we then have

$$
k_{1} \leq\|w\|_{g_{c, \Phi}} \leq k_{2}
$$

Let $W$ be the $g_{c, \Phi}$-parallel transport of $w$ along $\gamma$ and let $\left\{E_{1}, E_{2}, E_{3}\right\}$ be any $g_{c, \Phi}$-parallel orthonormal frame along $\gamma$. Since the Christoffel symbols of $g_{c, \Phi}$ are controlled by $m /\left(\sigma_{i}+c\right) \leq 1$ with all their derivatives and since $\|w\|_{\delta}=1$, there is a time of existence $T^{\prime}$ (which is independent of our choices of $v$ and $w$ ) such that on $\left[0, T^{\prime}\right]$ we have

$$
\|w-W\|_{\delta} \leq \frac{1}{4}
$$

For $1 \leq i \leq 3$ define the auxiliary functions

$$
F_{i}(t)=g_{c, \Phi}\left(Y-t W, E_{i}\right) .
$$

Note that $F_{i}(0)=F_{i}^{\prime}(0)=0$. The Jacobi equation implies

$$
\begin{aligned}
F_{i}^{\prime \prime}(t) & =-R_{g_{c, \Phi}}\left(Y, \gamma^{\prime}, \gamma^{\prime}, E_{i}\right) \\
& =-R_{g_{c, \Phi}}\left(Y-t W, \gamma^{\prime}, \gamma^{\prime}, E_{i}\right)-t R_{g_{c, \Phi}}\left(W, \gamma^{\prime}, \gamma^{\prime}, E_{i}\right) .
\end{aligned}
$$

Temporarily fix a value of $0<t_{0}<T$. By Proposition 3.7 the sectional curvatures of $g_{c, \Phi}$ are controllable by $m /\left(\sigma_{i}+c\right)<1$, and so the same applies to the Jacobi operators $R_{g_{c, \Phi}}\left(., \gamma^{\prime}\right) \gamma^{\prime}$. In particular, the fact that $\left\|E_{i}\right\|_{g_{c, \Phi}}=1$ and $\|W\|_{g_{c, \Phi}} \leq k_{2}$ along $\gamma(t)$, implies

$$
\left|F_{i}^{\prime \prime}(t)\right| \leq \kappa\left(\sup _{t \leq t_{0}}\|Y-t W\|_{g_{c, \Phi}}+k_{2} t\right), \quad 0 \leq t \leq t_{0}
$$

where $\kappa$ denotes the bound on the norms of the Jacobi operators $R_{g_{c, \Phi}}\left(., \gamma^{\prime}, \gamma^{\prime}, E_{i}\right)$. Upon integration we obtain

$$
\left|F_{i}(t)\right| \leq \kappa\left(t^{2}\left(\sup _{t \leq t_{0}}\|Y-t W\|_{g_{c, \Phi}}\right)+\frac{1}{3} k_{2} t^{3}\right) .
$$

As $\|Y-t W\|_{g_{c, \Phi}} \leq \sum_{i=1}^{3}\left|F_{i}(t)\right|$ we obtain

$$
\|Y-t W\|_{g_{c, \Phi}} \leq 3 \kappa\left(t^{2}\left(\sup _{t \leq t_{0}}\|Y-t W\|_{g_{c, \Phi}}\right)+\frac{1}{3} k_{2} t^{3}\right) .
$$


Under the assumption of $1-3 \kappa t_{0}^{2} \geq \frac{1}{2}$, i.e $t_{0} \leq \frac{1}{\sqrt{6 \kappa}}$, and after taking the supremum over $0 \leq t \leq t_{0}$, we arrive at

$$
\sup _{t \leq t_{0}}\|Y-t W\|_{g_{c, \Phi}} \leq 2 \kappa k_{2} t_{0}^{3}
$$

Since

$$
\left\|Y-t_{0} W\right\|_{\delta} \leq \sup _{t \leq t_{0}}\|Y-t W\|_{\delta} \leq \frac{1}{k_{1}} \sup _{t \leq t_{0}}\|Y-t W\|_{g_{c, \Phi}}
$$

and since $t_{0}<T$ was arbitrary we see that

$$
\|Y-t W\|_{\delta} \leq 2 \kappa \frac{k_{2}}{k_{1}} t^{3} \text { i.e }\left\|\frac{1}{t} Y(t)-W\right\|_{\delta}<2 \kappa \frac{k_{2}}{k_{1}} t^{2}
$$

for all $0 \leq t<\min \left\{\frac{1}{\sqrt{6 \kappa}}, T\right\}$. If in addition $t<T^{\prime}$, we have

$$
\left\|w-\frac{1}{t} Y(t)\right\|_{\delta} \leq 2 \kappa \frac{k_{2}}{k_{1}} t^{2}+\frac{1}{4} .
$$

It follows that so long as $\|v\|_{g_{c, \Phi}}$ is such that

$$
0 \leq\|v\|_{g_{c, \Phi}}<\min \left\{\frac{1}{\sqrt{6 \kappa}}, T, T^{\prime}\right\}
$$

and

$$
2 \kappa \frac{k_{2}}{k_{1}}\|v\|_{g_{c, \Phi}}^{2}<\frac{1}{4}
$$

the estimate (3.58) is fulfilled. The boundedness of $\frac{k_{2}}{k_{1}}$ (see Proposition 3.7) implies that the estimate (3.58) holds for all $v \in B_{g_{c, \Phi}}\left(Q, 2 r_{0}\right)$ where $r_{0}$ can be chosen independently of $Q$. This completes our proof.

\subsection{The Area of the Minimal Surface}

Here we use the estimates in the prior subsection combined with ColdingMinicozzi's monotonicity formula for the area of a minimal surface to prove the following theorem depicted in Figure 3.1.

Theorem 3.13. Fix a length $c>0$. Let $(M, g)$ be a geometrostatic manifold such that for all $i=1,2, \ldots, n$ we have

$$
\frac{m}{\sigma_{i}+c} \leq 1
$$

and

$$
\sigma_{i}>5 c \quad \text { or } \quad \sigma_{i}^{\text {out }}<\frac{c}{5} .
$$

Consider the scaled pullback metric $g_{c, \Phi}$ on the Euclidean annulus, $\mathscr{A}$, as defined in (3.36) 3.37). Then there is an $s_{0}>0$ which is independent of the choice of our geometrostatic manifold satisfying the conditions above such that for any smooth connected $g_{c, \Phi}$-minimal surface $\Sigma$ in $\mathscr{A}$ with

$$
\partial \Sigma \subseteq \partial B_{\delta}(0,2) \cup \partial B_{\delta}(0,1)
$$


that contain points

$$
q \in \partial \Sigma \cap \partial B_{\delta}(0,2) \text { and } q^{\prime} \in \partial \Sigma \cap \partial B_{\delta}(0,1)
$$

must satisfy

$$
\operatorname{Area}_{g_{c, \Phi}}(\Sigma) \geq\left(\pi e^{-2}\right) s_{0}^{2}
$$

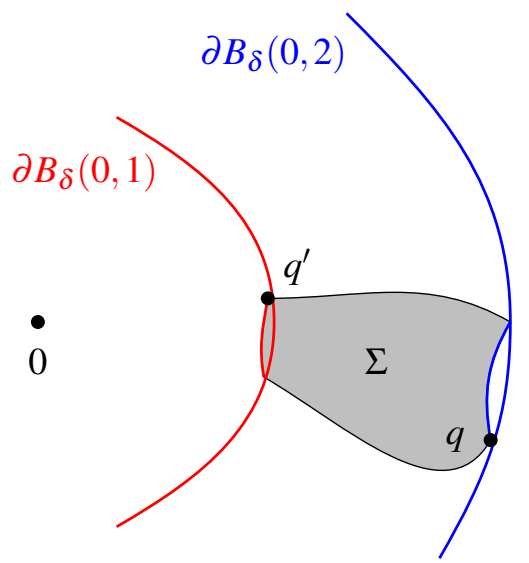

FIGURE 3.1. The minimal surface, $\Sigma$, of Theorem 3.13

Before we prove the theorem we recall the following monotonicity formula (7.5) from Colding \& Minicozzi's textbook [CM11].

Theorem 3.14 (Monotonicity Formula). Let $x_{0}$ be a point on a smooth minimal surface $\Sigma$ in a 3-manifold $(M, g)$. Let $\kappa>0$ be a bound on sectional curvatures $K_{M}$ on $M$ (as in $\left|K_{M}\right|<\kappa$ ) and let $i_{0}>0$ denote a positive lower bound on the injectivity radius on $M$. Then the function

$$
e^{2 \sqrt{\kappa} s} s^{-2} \operatorname{Area}\left(B_{s, g}\left(x_{0}\right) \cap \Sigma\right)
$$

of $0<s<\min \left\{i_{0}, \frac{1}{\sqrt{\kappa}}, \operatorname{dist}\left(x_{0}, \partial \Sigma\right)\right\}$ is non-decreasing.

For smooth minimal surfaces the function in Theorem 3.14 converges to $\pi$ as $s \rightarrow 0$. Consequently, the monotonicity formula gives us an inequality of the form

$$
\operatorname{Area}\left(B_{s, g}\left(x_{0}\right) \cap \Sigma\right) \geq\left(\pi e^{-2 \sqrt{\kappa} s}\right) s^{2} \geq\left(\pi e^{-2}\right) s^{2}
$$

on the interval for $s$ stated in the theorem.

Following an idea used by Jauregui in [Jau14], we can use this monotonicity formula to provide a lower bound for the area of a minimal surface which runs between two spheres as follows:

Proposition 3.15. Let $g \geq \delta$ be a metric on $B_{\delta}(0,4) \backslash B_{\delta}\left(0, \frac{1}{2}\right)$ whose sectional curvatures are bounded by $\kappa$ and whose injectivity radii on $B_{\delta}(0,2) \cup \partial B_{\delta}(0,1)$ are 
bounded from below by $i_{0}$. Let $s_{0}=\frac{1}{2} \min \left\{i_{0}, \frac{1}{\sqrt{\kappa}}, \frac{1}{3}\right\}$. Then all smooth connected minimal surfaces $\Sigma$ with

$$
\partial \Sigma \subseteq \partial B_{\delta}(0,2) \cup \partial B_{\delta}(0,1)
$$

that contain points

$$
q \in \partial \Sigma \cap \partial B_{\delta}(0,2) \text { and } q^{\prime} \in \partial \Sigma \cap \partial B_{\delta}(0,1)
$$

must satisfy

$$
\operatorname{Area}_{g}(\Sigma) \geq\left(\pi e^{-2}\right) s_{0}^{2}
$$

Proof. Take $x_{0} \in \Sigma \cap \partial B_{\delta}(0,3 / 2)$ which exists because $\Sigma$ is connected and has points $q$ and $q^{\prime}$ as in the hypothesis. Since $g \geq \delta$

$$
B_{g}\left(x_{0}, 1 / 2\right) \subset B_{\delta}\left(x_{0}, 1 / 2\right) \subset B_{\delta}(0,1) \backslash B_{\delta}(0,2) .
$$

Since $s_{0}<1 / 2$ and satisfies the given bounds depending on injectivity radius and sectional curvature, we can apply Theorem 3.14 and (3.81) to obtain

$$
\operatorname{Area}_{g}(\Sigma) \geq \operatorname{Area}_{g}\left(\Sigma \cap B_{g}\left(x_{0}, s_{0}\right)\right) \geq\left(\pi e^{-2}\right) s_{0}^{2} .
$$

We now prove Theorem 3.13 .

Proof of Theorem 3.13 Note that $c$ in Theorem 3.13 satisfies (3.34) which is the hypothesis of Propositions 3.7 and 3.8. So we obtain uniform bounds on $i_{0}$ and $\kappa$ for all $(M, g)$. Thus the value of $s_{0}$ in Proposition 3.15 applied to the metric $g_{c, \Phi_{0}}=$ $c^{-2} \Phi_{0}^{*} g$ does not depend on $(M, g)$ satisfying the hypotheses of the theorem.

\subsection{Part of the Minimal Surface is near the Point}

Before we prove Theorem 1.2 we prove that every outermost minimal surface intersects with a small ball about one of the $p_{i} \in P$. Lemma 3.16 is applied to show each $\Sigma_{i}$ for $i=1 . . n$ intersects with a small ball about $p_{i}$. Lemma3.18 can be applied to every outermost minimal surface, even the ones which do not surround a $p_{i}$.

Lemma 3.16. In any Riemannian manifold, $M \subseteq \mathbb{R}^{3}$, endowed with a metric $g \geq \delta$, a surface $\Sigma^{\prime}=\partial \Omega$ surrounding a point $p \in \Omega$ satisfies

$$
\Sigma^{\prime} \cap \bar{B}_{\delta}\left(p, \sqrt{\operatorname{Area}_{g}\left(\Sigma^{\prime}\right) /(4 \pi)}\right) \neq \emptyset .
$$

Proof. Indeed, had there existed a $v>1$ such that

$$
\bar{B}_{\delta}\left(p, v \sqrt{\operatorname{Area}_{g}\left(\Sigma^{\prime}\right) /(4 \pi)}\right) \subset \Omega .
$$


then we would have

(3.89) $\operatorname{Area}_{g}\left(\Sigma^{\prime}\right) \geq \operatorname{Area}_{\delta}\left(\Sigma^{\prime}\right)$ because $g \geq \delta$,

$$
\begin{aligned}
& \geq C_{\text {iso }}\left(\operatorname{Vol}_{\delta}(\Omega)\right)^{2 / 3} \text { by the isoperimetric inequality, } \\
& \geq C_{\text {iso }}\left(\operatorname{Vol}_{\delta}\left(B_{\delta}\left(p, v \sqrt{\operatorname{Area}_{g}\left(\Sigma^{\prime}\right) /(4 \pi)}\right)\right)\right)^{2 / 3} \text { by (3.88), }
\end{aligned}
$$$$
=\operatorname{Area}_{\delta}\left(\partial B_{\delta}\left(p, v \sqrt{\operatorname{Area}_{g}\left(\Sigma^{\prime}\right) /(4 \pi)}\right)\right)
$$$$
=4 \pi\left(v \sqrt{\operatorname{Area}_{g}\left(\Sigma^{\prime}\right) /(4 \pi)}\right)^{2}=v^{2} \operatorname{Area}_{g}\left(\Sigma^{\prime}\right)>\operatorname{Area}_{g}\left(\Sigma^{\prime}\right),
$$

which is a contradiction.

Remark 3.17. By taking $p=p_{i}$ we see that in the cases of the outermost minimal surfaces $\Sigma_{i}$ surrounding $p_{i}$ we have

$$
\Sigma_{i} \cap \bar{B}_{\delta}\left(p_{i}, \sqrt{\operatorname{Area}_{g}\left(\Sigma_{i}\right) /(4 \pi)}\right) \neq \emptyset .
$$

As it is possible there are other outermost minimizing surfaces which do not contain a point $p_{i}$, we prove the following lemma using the area lower bounds developed in Proposition 3.2.

Lemma 3.18. Let $\Sigma_{i}$ be an outermost minimal surface of a geometrostatic manifold, $-n^{\prime} \leq i \leq n$. There exists $j=j(i) \in\{1, \ldots, n\}$ and $p_{j} \in P$ with

$$
\Sigma_{i} \cap B_{\delta}\left(p_{j}, \sqrt{\operatorname{Area}_{g}\left(\Sigma_{i}\right) / \pi}\right) \neq \emptyset .
$$

Note that $j(i)=i$ when $i \geq 1$.

Proof. Suppose the opposite: that for all $p_{j} \in P$ we have

$$
\Sigma_{i} \cap B_{\delta}\left(p_{j}, \sqrt{\operatorname{Area}_{g}\left(\Sigma_{i}\right) / \pi}\right)=\emptyset .
$$

This is true iff for all $p_{j} \in P$ we have

$$
\frac{1}{\left|x-p_{j}\right|} \leq \sqrt{\frac{\pi}{\operatorname{Area}\left(\Sigma_{i}\right)}}
$$

for all $x \in \Sigma_{i}$. By the work of Huisken-Ilmanen $\Sigma_{i}$ is diffeomorphic to $S^{2}$, and so Proposition 3.2 implies

$$
\sqrt{\frac{4 \pi}{\text { Area }_{g}\left(\Sigma_{i}\right)}} \leq \max _{x \in \Sigma_{i}}\left\{\frac{\left|\nabla \Psi_{x}\right|}{\Psi^{2}(x)}\right\}
$$


where $\Psi(x)=\chi(x) \psi(x)$. We proceed by estimating $\left|\nabla \Psi_{x}\right| / \Psi^{2}(x)$ using (3.97):

$$
\frac{\left|\nabla \Psi_{x}\right|}{\Psi^{2}(x)} \leq \frac{\left|\nabla \Psi_{x}\right|}{\Psi(x)} \leq \frac{\left|\nabla \chi_{x}\right|}{\chi(x)}+\frac{\left|\nabla \psi_{x}\right|}{\psi(x)}
$$

$$
\begin{aligned}
& \leq \frac{\left(\sum_{j} \frac{\alpha_{j}}{\left|x-p_{j}\right|^{2}}\right)}{\chi(x)}+\frac{\left(\sum_{j} \frac{\beta_{j}}{\left|x-p_{j}\right|^{2}}\right)}{\psi(x)} \\
& \leq \sum_{j} \frac{1}{\left|x-p_{j}\right|}\left(\frac{\left(\frac{\alpha_{j}}{\left|x-p_{j}\right|}\right)}{\chi(x)}+\frac{\left(\frac{\beta_{j}}{\left|x-p_{j}\right|}\right)}{\psi(x)}\right)
\end{aligned}
$$$$
\leq \sqrt{\frac{\pi}{\operatorname{Area}_{g}\left(\Sigma_{i}\right)}}\left(\frac{\left(\sum_{j} \frac{\alpha_{j}}{\left|x-p_{j}\right|}\right)}{\chi(x)}+\frac{\left(\sum_{j} \frac{\beta_{j}}{\left|x-p_{j}\right|}\right)}{\psi(x)}\right)
$$

$$
=\sqrt{\frac{\pi}{\operatorname{Area}_{g}\left(\Sigma_{i}\right)}}\left(\frac{(\chi(x)-1)}{\chi(x)}+\frac{(\psi(x)-1)}{\psi(x)}\right)<2 \sqrt{\frac{\pi}{\text { Area }_{g}\left(\Sigma_{i}\right)}} .
$$

This chain of inequalities proves that

$$
\max _{x \in \Sigma_{i}}\left\{\frac{\left|\nabla \Psi_{x}\right|}{\Psi^{2}(x)}\right\}<\sqrt{\frac{4 \pi}{\text { Area }_{g}\left(\Sigma_{i}\right)}}
$$

which is a direct contradiction to (3.98). The final note follows from Lemma 3.16.

\subsection{The Whole Minimal Surface is Near the Point}

In this subsection we prove the first part of Theorem 1.2. Recall that $\Sigma_{i}$ for $-n^{\prime} \leq i \leq n$ denotes the $i^{t h}$ outermost minimal surface, and recall that

$$
\sigma_{j}=\min _{k \neq j}\left\{\left|p_{k}-p_{j}\right|\right\}, \sigma=\min _{j}\left\{\sigma_{j},\left|p_{j}\right|\right\} \text { and } \sigma_{j}^{\text {out }}=\max _{k \neq j}\left\{\left|p_{k}-p_{j}\right|\right\} \text {. }
$$

Proposition 3.19. Let $s_{0}$ be as in the Theorem 3.13. The universal constant

$$
C_{1}=1+2 e / s_{0}
$$

is such that for all geometrostatic $\left(\mathbb{R}^{3} \backslash P, g\right)$ with $\sigma>20 m C_{1}$ and all $-n^{\prime} \leq i \leq n$ there is a $j=j(i) \in\{1, \ldots, n\}$ and $p_{j} \in P$ with

$$
\Sigma_{i} \subseteq B_{\delta}\left(p_{j}, 2 C_{1} \sqrt{\operatorname{Area}_{g}\left(\Sigma_{i}\right) / \pi}\right) .
$$

Furthermore, if $i>0$ then we may take $j(i)=i$.

This proposition is a consequence of the following lemma which will be applied again later in the paper as well:

Lemma 3.20. Let $s_{0}$ be as in the Theorem 3.13, let $C_{1}=1+2 e / s_{0}$. 
- CASE 1: Suppose $1 \leq i \leq n$ and suppose that $r \geq \sqrt{\operatorname{Area}_{g}\left(\Sigma_{i}\right) / \pi}$ is a radius such that

$$
\sigma_{i}>5 C_{1} r \text { or } \sigma_{i}^{\text {out }}<\frac{1}{5} C_{1} r
$$

We then have

$$
\Sigma_{i} \subseteq B_{\delta}\left(p_{i}, 2 C_{1} r\right)
$$

- CASE 2: Suppose that $-n^{\prime} \leq i \leq 0$ and suppose that $r \geq \sqrt{\operatorname{Area}_{g}\left(\Sigma_{i}\right) / \pi}$ is a radius such that

$$
\sigma>5 C_{1} r
$$

Then there is a $j=j(i) \in\{1, \ldots, n\}$ and $p_{j} \in P$ for which

$$
\Sigma_{i} \subseteq B_{\delta}\left(p_{j}, 2 C_{1} r\right) .
$$

Before proving Lemma 3.20, we apply it to prove Proposition 3.19.

Proof of Proposition 3.19 We are given $\sigma>20 m C_{1}$. Let $r=\sqrt{\operatorname{Area}_{g}\left(\Sigma_{i}\right) / \pi}$. Then by the Penrose inequality:

$$
\sigma>20 C_{1} m \geq 20 C_{1} \sqrt{\operatorname{Area}_{g}\left(\Sigma_{i}\right) /(16 \pi)}=5 C_{1} r .
$$

Such an $r$ satisfies $r<\sigma /\left(5 C_{1}\right)$, so we have (3.109) which implies (3.107).

We now prove Lemma 3.20.

Proof of Lemma 3.20 Let $j=j(i)$ be as in Remark 3.17) and Lemma 3.18. Suppose the opposite: there exists a point,

$$
q_{i} \in \Sigma_{i} \backslash B_{\delta}\left(p_{j}, 2 C_{1} r\right) .
$$

Applying Lemma 3.18 we are able to conclude that $\Sigma_{i}$ contains a point

$$
q_{i} \in B_{\delta}\left(p_{j}, C_{1} \sqrt{\operatorname{Area}_{g}\left(\Sigma_{i}\right) / \pi}\right) \subseteq B_{\delta}\left(p_{j}, C_{1} r\right) .
$$

Since $\Sigma_{i}$ is closed and connected, this means we can choose $q_{i}$ above such that

$$
q_{i} \in \Sigma_{i} \cap \partial B_{\delta}\left(p_{j}, 2 C_{1} r\right)
$$

and we can choose

$$
q_{i}^{\prime} \in \Sigma_{i} \cap \partial B_{\delta}\left(p_{j}, C_{1} r\right) .
$$

In particular the minimal surface depicted in Figure 3.2,

$$
S_{i}=\Sigma_{i} \cap\left(\bar{B}_{\delta}\left(p_{j}, 2 C_{1} r\right) \backslash B_{\delta}\left(p_{j}, C_{1} r\right)\right),
$$

contains the points, $q_{i}$ and $q_{i}^{\prime}$, as above.

Consider the embedding

$$
\Phi:\left(B_{\delta}(0,4) \backslash B_{\delta}\left(0, \frac{1}{4}\right)\right) \rightarrow \mathbb{R}^{3} \backslash P \text { given by } u \mapsto p_{j}+c_{1} u
$$

where $c_{1}=C_{1} r$. 


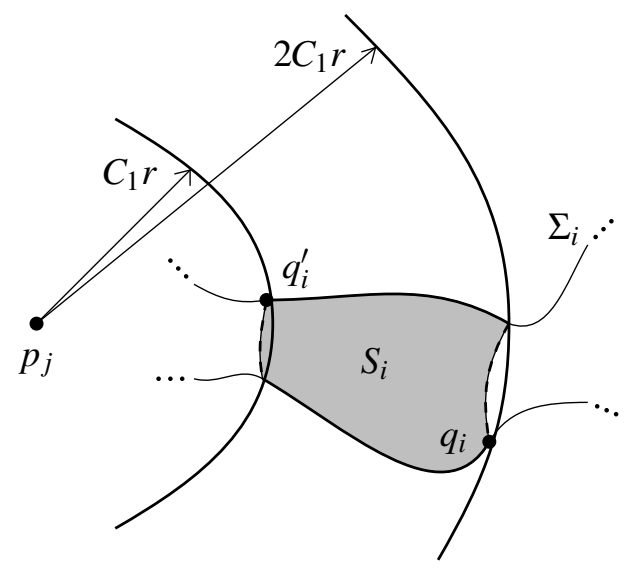

FIGURE 3.2. The minimal surface, $S_{i} \subset \Sigma_{i}$.

The surface $\Phi^{-1}\left(S_{i}\right)$ is minimal with respect to $g_{c_{1}, \Phi}$ and has points

$$
\Phi^{-1}\left(q_{i}\right) \in \Phi^{-1}\left(S_{i}\right) \cap \Phi^{-1}\left(\partial B_{\delta}\left(p_{i}, 2 C_{1} r\right)\right)=\Phi^{-1}\left(S_{i}\right) \cap \partial B_{\delta}(0,2)
$$

and

$$
\Phi^{-1}\left(q_{i}^{\prime}\right) \in \Phi^{-1}\left(S_{i}\right) \cap \Phi^{-1}\left(\partial B_{\delta}\left(p_{i}, C_{1} r\right)\right)=\Phi^{-1}\left(S_{i}\right) \cap \partial B_{\delta}(0,1) .
$$

In addition,

$$
\partial \Phi^{-1}\left(S_{i}\right) \subset \partial B_{\delta}(0,2) \cup \partial B_{\delta}(0,1)
$$

We may now apply Theorem 3.13 to obtain

$$
\operatorname{Area}_{g_{c_{1}, \Phi}}\left(\Phi^{-1}\left(S_{i}\right)\right) \geq \pi e^{-2} s_{0}^{2}
$$

Thus
(3.123) $\operatorname{Area}_{g}\left(\Sigma_{i}\right) \geq \operatorname{Area}_{g}\left(S_{i}\right)$
by $S_{i} \subset \Sigma_{i}$
$=\operatorname{Area}_{\Phi^{*} g}\left(\Phi^{-1}\left(S_{i}\right)\right)$ by the defn of pullback $=c_{1}^{2} \operatorname{Area}_{g_{c_{1}, \Phi}}\left(\Phi^{-1}\left(S_{i}\right)\right)$ by rescaling $\geq c_{1}^{2} \pi e^{-2} s_{0}^{2}$ by (3.79) $=\left(C_{1} x\right)^{2} \pi e^{-2} s_{0}^{2}$ by $c_{1}=C_{1} r$ $\geq s_{0}^{2} C_{1}^{2} \operatorname{Area}_{g}\left(\Sigma_{i}\right) / e^{2}$ $>\operatorname{Area}_{g}\left(\Sigma_{i}\right)$

$$
\begin{aligned}
& \text { by } r \geq \sqrt{\operatorname{Area}_{g}\left(\Sigma_{i}\right) / \pi} \\
& \text { because } C_{1}>e / s_{0},
\end{aligned}
$$

which is a contradiction. 


\subsection{The Minimal Surface is Not Too Close to the Point}

We now prove the second part of Theorem 1.2. Recall $C_{1}$ defined in Proposition 3.19 .

Proposition 3.21. If $m<\sigma /\left(20 C_{1}\right)$, then

$$
\Sigma_{i} \cap B_{\delta}\left(p_{i}, \frac{\alpha_{i} \beta_{i}}{4 C_{1}\left(\alpha_{i}+\beta_{i}\right)}\right)=\emptyset \text { for all } 1 \leq i \leq n .
$$

In order to prove Proposition 3.21 we apply an inversion to the geometrostatic manifold sending $p_{i}$ to $\infty$. As we could not find this inversion process in the literature, we provide the details in the appendix. See Theorem A.1.

Proof of Proposition 3.21 Without loss of generality take $i=n$. Apply the inversion of Theorem A.1 with $x_{i}=p_{i}$, to obtain a minimal surface $F^{-1}\left(\Sigma_{n}\right)$ about $y_{0}=0$ in

$$
Y=\mathbb{R}^{n} \backslash\left\{y_{0}, \ldots, y_{n-1}\right\} \text { with } g_{Y}=\left(1+\sum_{i=0}^{n-1} \frac{\alpha_{Y, i}}{\left|y-y_{i}\right|}\right)^{2}\left(1+\sum_{i=0}^{n-1} \frac{\beta_{Y, i}}{\left|y-y_{i}\right|}\right)^{2} \delta_{y} .
$$

There is an outermost minimal surface about $y_{0}=0$ such that

$$
\Sigma_{0}=\partial \Omega_{0} \subset Y \text {. }
$$

By the definition of outermost as in Section 3.1, we know

$$
F^{-1}\left(\Sigma_{n}\right) \subset \Omega_{0} .
$$

Let

$$
A_{0}=\operatorname{Area}_{g_{Y}}\left(\Sigma_{0}\right)
$$

In Corollary A.2 it was seen that the ADM mass of $\left(Y, g_{Y}\right)$ is

$$
m_{Y}=\alpha_{n}+\beta_{n}+\sum_{j=1}^{n-1} \frac{\alpha_{n} \beta_{j}+\alpha_{j} \beta_{n}}{r_{j, n}}=m_{n}
$$

Observe that

$$
m_{n} \leq \alpha_{n}+\beta_{n}+\alpha_{n} \sum_{j=1}^{n-1} \frac{\beta_{j}}{\sigma}+\beta_{n} \sum_{j=1}^{n-1} \frac{\alpha_{j}}{\sigma}<\left(\alpha_{n}+\beta_{n}\right)\left(1+\frac{m}{\sigma}\right) \leq 2\left(\alpha_{n}+\beta_{n}\right)
$$

By the Penrose Inequality, and the fact that $\Sigma_{0}$ is outermost minimizing in $\left(Y, g_{Y}\right)$ we have

$$
m_{n}=m_{Y} \geq \sqrt{A_{0} /(16 \pi)} .
$$

In addition, by Theorem A.1, the $0^{t h}$ separation constant of $\left(Y, g_{Y}\right)$ satisfies

$$
\sigma_{0}^{\text {out }}=\max _{j \neq 0}\left\{\left|y_{j}-0\right|\right\}=\max _{j \neq 0}\left\{\alpha_{n} \beta_{n} / r_{j, n}\right\} \leq \alpha_{n} \beta_{n} / \sigma
$$


where $\sigma$ is the separation constant for $M$ as in (1.7). Furthermore

$$
\frac{\alpha_{n} \beta_{n}}{\sigma}=\frac{\alpha_{n}}{\left(\alpha_{n}+\beta_{n}\right)} \frac{\beta_{n}}{\sigma}\left(\alpha_{n}+\beta_{n}\right) \leq 1 \cdot \frac{m}{\sigma}\left(\alpha_{n}+\beta_{n}\right) \leq \frac{1}{5}\left(\alpha_{n}+\beta_{n}\right) .
$$

In particular

$$
\sigma_{0}^{\text {out }} \leq m_{n} / 5
$$

We may apply Lemma3.20 to the geometrostatic manifold $\left(Y, g_{Y}\right)$ with $i=0$ taking $r=m_{n}$ by (3.138) and (3.140). Thus

$$
\Sigma_{0} \subseteq B_{\delta}\left(0,2 C_{1} r\right)
$$

As a consequence, by (3.133), we have

$$
F^{-1}\left(\Sigma_{n}\right) \subset \Omega_{0} \subseteq B_{\delta}\left(0,2 C_{1} r\right) .
$$

Thus

$$
\Sigma_{n} \subset F\left(B_{\delta}\left(0,2 C_{1} r\right)\right)=\mathbb{R}^{3} \backslash \bar{B}_{\delta}\left(p_{n}, \frac{\alpha_{n} \beta_{n}}{2 C_{1} r}\right)
$$

By our choice of $r=m_{n}$ and (3.136) we have

$$
r<2\left(\alpha_{n}+\beta_{n}\right)
$$

which implies that

$$
\frac{\alpha_{n} \beta_{n}}{2 C_{1} r}>\frac{\alpha_{n} \beta_{n}}{4 C_{1}\left(\alpha_{n}+\beta_{n}\right)}
$$

Thus

$$
\Sigma_{n} \cap B_{\delta}\left(p_{n}, \frac{\alpha_{n} \beta_{n}}{4 C_{1}\left(\alpha_{n}+\beta_{n}\right)}\right) \subset \Sigma_{n} \cap B_{\delta}\left(p_{n}, \frac{\alpha_{n} \beta_{n}}{2 C_{1} r}\right)=\emptyset,
$$

and our proof is now complete.

\subsection{Proof of Theorems 1.2 and 1.4}

Theorem 1.2 is a special case of the following, more general result. The result shows that all the outermost minimal surfaces $\Sigma_{i}$ with $i \in\left\{-n^{\prime}, \ldots, n\right\}$ are located in an annular neighborhood of some point $p_{j(i)} \in P$.

Theorem 3.22. Let $s_{0}$ be as in the Theorem 3.13, The universal constant

$$
C_{1}=1+2 e / s_{0}
$$

is such that for all geometrostatic $\left(\mathbb{R}^{3} \backslash P, g\right)$ with $\sigma>20 m C_{1}$ and all $-n^{\prime} \leq i \leq n$ there is a $j=j(i) \in\{1, \ldots, n\}$ and $p_{j} \in P$ with

$$
\Sigma_{i} \subseteq B_{\delta}\left(p_{j}, 2 C_{1} \sqrt{\operatorname{Area}_{g}\left(\Sigma_{i}\right) / \pi}\right) \backslash B_{\delta}\left(p_{j}, \frac{\alpha_{j} \beta_{j}}{4 C_{1}\left(\alpha_{j}+\beta_{j}\right)}\right) .
$$

Furthermore, if $i>0$ then we may take $j(i)=i$. 
Proof. Note that for all $i \in\left\{-n^{\prime}, \ldots, n\right\}$ there is a $j=j(i) \in\{1, \ldots, n\}$ and $p_{j} \in P$ with

$$
\Sigma_{i} \subseteq B_{\delta}\left(p_{j}, 2 C_{1} \sqrt{\operatorname{Area}_{g}\left(\Sigma_{i}\right) / \pi}\right)
$$

by Proposition 3.19. Furthermore for $j \in\{1, \ldots, n\}$ by Proposition 3.21 we have

$$
\Sigma_{j} \cap B_{\delta}\left(p_{j}, \frac{\alpha_{j} \beta_{j}}{4 C_{1}\left(\alpha_{j}+\beta_{j}\right)}\right)=\emptyset
$$

so

$$
B_{\delta}\left(p_{j}, \frac{\alpha_{j} \beta_{j}}{4 C_{1}\left(\alpha_{j}+\beta_{j}\right)}\right) \subset \Omega_{j} .
$$

We see from (3.4) that $\Sigma_{j} \cap \Omega_{j(i)}=\emptyset$ for all $i \in\left\{-n^{\prime}, \ldots, 0\right\}$. Combining this observation with (3.151) yields

$$
\Sigma_{i} \cap B_{\delta}\left(p_{j}, \frac{\alpha_{j} \beta_{j}}{4 C_{1}\left(\alpha_{j}+\beta_{j}\right)}\right)=\Sigma_{i} \cap \Omega_{j}=\emptyset,
$$

which completes our proof.

For $j \in\{1, \ldots, n\}$ define

$$
I(j):=\left\{i \mid-n^{\prime} \leq i \leq n, \Sigma_{i} \subseteq B_{\delta}\left(p_{j}, 2 C_{1} \sqrt{\operatorname{Area}_{g}\left(\Sigma_{i}\right) / \pi}\right)\right\} .
$$

Loosely speaking, the set $I(j)$ identifies those outermost minimal surfaces which are close to one of $p_{j} \in P$. Next, introduce

$$
\gamma_{j}:=\max \left\{2 C_{1} \sqrt{\operatorname{Area}_{g}\left(\Sigma_{i}\right) / \pi} \mid i \in I(j)\right\}
$$

and note that

$$
\gamma_{j} \leq 8 C_{1} m .
$$

by the Penrose inequality. The lengths $\gamma_{j}$ are the radii within which all the outermost minimal surfaces are to be found. With this notation established, Theorem 1.4 is an immediate corollary of Theorem 3.22 .

\section{Almost Rigidity of the Positive Mass Theorem}

In this section we prove the Almost Rigidity of the Positive Mass Theorem for geometrostatic manifolds [Theorem 1.5]. Observe that our result includes (but is not limited to) geometrostatic manifolds with a uniform upper bound on the number of black holes whose ADM mass is converging to 0 . 


\subsection{A Review of Intrinsic Flat Convergence}

The intrinsic flat distance, $d_{\mathscr{F}}$ between pairs of compact oriented Riemannian manifolds with boundary was first introduced by the first author with Wenger in [SW11]. Their intrinsic flat distance, like the classical flat distance of Geometric Measure Theory, does not scale well: the distance between two $n$ dimensional oriented manfolds is a sum of an $(n+1)$ dimensional filling volume and an $n$ dimensional volume. In joint work of the first author with LeFloch [LS15], the D-flat distance $d_{D \mathscr{F}}$ was defined, dividing the $(n+1)$ dimensional volume by diameter before adding it the $n$ dimensional volume.

In work of Lakzian and the first author [LS13] the following theorem was proven providing a concrete means to estimate the intrinsic flat distance. Here we state it also adding in the estimate on the D-flat distance multiplied by diameter:

Theorem 4.1. Suppose $\left(M_{1}, g_{1}\right)$ and $\left(M_{2}, g_{2}\right)$ are oriented precompact Riemannian manifolds with diffeomorphic subregions $W_{i} \subset M_{i}$. Identifying $W_{1}=W_{2}=W$ assume that on $W$ we have

$$
g_{1} \leq(1+\varepsilon)^{2} g_{2} \text { and } g_{2} \leq(1+\varepsilon)^{2} g_{1} .
$$

Taking the extrinsic diameters,

$$
\operatorname{diam}\left(M_{i}\right) \leq D
$$

we define a hemispherical width,

$$
a>\frac{\arccos (1+\varepsilon)^{-1}}{\pi} D .
$$

Taking the difference in distances with respect to the outside manifolds, we set

$$
\lambda=\sup _{x, y \in W}\left|d_{M_{1}}(x, y)-d_{M_{2}}(x, y)\right| \leq 2 D,
$$

and we define the height,

$$
\bar{h}=\max \left\{\sqrt{2 \lambda D}, D \sqrt{\varepsilon^{2}+2 \varepsilon}\right\} .
$$

Then

$$
\begin{aligned}
d_{\mathscr{F}}\left(M_{1}, M_{2}\right) \leq & (2 \bar{h}+a)\left(\operatorname{Vol}_{m}\left(W_{1}\right)+\operatorname{Vol}_{m}\left(W_{2}\right)+\operatorname{Vol}_{m-1}\left(\partial W_{1}\right)+\operatorname{Vol}_{m-1}\left(\partial W_{2}\right)\right) \\
& +\operatorname{Vol}_{m}\left(M_{1} \backslash W_{1}\right)+\operatorname{Vol}_{m}\left(M_{2} \backslash W_{2}\right),
\end{aligned}
$$

and

$$
\begin{aligned}
D d_{D \mathscr{F}}\left(M_{1}, M_{2}\right) \leq & (2 \bar{h}+a)\left(\operatorname{Vol}_{m}\left(W_{1}\right)+\operatorname{Vol}_{m}\left(W_{2}\right)+D \operatorname{Vol}_{m-1}\left(\partial W_{1}\right)+D \operatorname{Vol}_{m-1}\left(\partial W_{2}\right)\right) \\
& +\operatorname{Vol}_{m}\left(M_{1} \backslash W_{1}\right) D+\operatorname{Vol}_{m}\left(M_{2} \backslash W_{2}\right) D .
\end{aligned}
$$




\subsection{Our strategy}

We start by fixing a geometrostatic manifold $(M, g)$ and a value of $R>0$ such that $\left|p_{i}\right| \neq R$ for all $1 \leq i \leq n$. We let

$$
M_{1}=\left\{x \in M^{\prime} \mid d_{\left(M^{\prime}, g\right)}(0, x)<R\right\}=B_{g}(0, R) \subseteq M^{\prime}
$$

endowed with the distance $d_{\left(M^{\prime}, g\right)}$ and

$$
M_{2}=\left\{x \in \mathbb{R}^{3}|| x \mid<R\right\}=B_{\delta}(0, R)
$$

endowed with the distance $d_{\left(\mathbb{R}^{3}, d_{\delta}\right)}(x, y)=|x-y|$. In the next few sections we prove estimates which allow us to apply Theorem 4.1. Ultimately, we obtain the following bound on the intrinsic flat distance between $M_{1}$ and $M_{2}$ with these distances defined above.

Proposition 4.2. There exist universal constants $\varepsilon_{0} \in(0,1), C_{\mathscr{F}}^{\prime}, C_{\mathscr{F}}^{\prime \prime}$ and $C_{D \mathscr{F}}$ such that for all $R>0$, all $\varepsilon \in\left(0, \varepsilon_{0}\right)$ and all Brill-Lindquist geometrostatic manifolds $(M, g)=\left(\mathbb{R}^{3} \backslash P,(\chi \psi)^{2} \delta\right)$ with

$$
m_{A D M}\left(M^{\prime}\right)=m<R \varepsilon^{3}, m<\varepsilon \cdot \frac{\sigma}{32} \quad \text { and } \rho(P) \cap(R-32 R \varepsilon, R+32 R \varepsilon)=\emptyset
$$

where $\rho(x)=|x|$ and $P=\left\{p_{1}, \ldots, p_{N}\right\}$, the intrinsic flat distance is estimated by

$$
d_{\mathscr{F}}\left(M_{1}, M_{2}\right) \leq C_{\mathscr{F}}^{\prime} R^{4} \sqrt{\varepsilon}+C_{\mathscr{F}}^{\prime \prime} R^{3} \sqrt{\varepsilon}
$$

and the D-flat distance is estimated by

$$
d_{D \mathscr{F}}\left(M_{1}, M_{2}\right) \leq C_{D \mathscr{F}} R^{3} \sqrt{\varepsilon} .
$$

Remark 4.3. Note that in Proposition 4.2 our estimates do not depend upon the number of points $p_{i} \in P$ nor on the number of minimal surfaces $\Sigma_{i}$.

The proof of Proposition 4.2 is involved and is proven in the next few subsections. The main result, Theorem 1.5, follows as a straightforward consequence of Proposition 4.2, see subsection 4.9 below for details.

\subsection{Locating $M_{1}$}

It is important to understand that there is a possibility for the asymptotic ends of $M$ to be connected by very long almost-cylindrical regions. In other words, there is a possibility for the connected components $\Sigma_{i}$ of $\partial M^{\prime}$ to be located very far down a deep well at $p_{i}$. See Example 2.5 where $\beta_{i}<<\alpha_{i}$.

In these settings $M_{1}=B_{g}(0, R) \subseteq M^{\prime}$ not only controls $|x|$ but also cuts off any long near-cylindrical regions near $p_{i}$. To make this idea precise we introduce the length

$$
\delta_{i, R}=\max \left\{\left(\alpha_{i}+\beta_{i}\right) \exp \left(\frac{-R}{\alpha_{i}+\beta_{i}}\right), \frac{\alpha_{i} \beta_{i}}{4 C_{1}\left(\alpha_{i}+\beta_{i}\right)}\right\} .
$$

When there is a long cylindrical neck then the first term achieves the maximum here. 
The next lemma clarifies how long near-cylindrical regions are cut off from $M_{1}$. Note that the material of this subsection is independent of our choice of $\varepsilon$.

Lemma 4.4. If $m<\sigma /\left(20 C_{1}\right)$ then

$$
M_{1} \subseteq B_{\delta}(0, R) \backslash\left(\bigcup_{i} B_{\delta}\left(p_{i}, \delta_{i, R}\right)\right) .
$$

Proof. First observe that since $g \geq \delta$ we have

$$
M_{1}=B_{g}(0, R) \subseteq B_{\delta}(0, R) .
$$

So we need only show that

$$
B_{g}(0, R) \subseteq \mathbb{R}^{3} \backslash\left(\bigcup_{i} B_{\delta}\left(p_{i}, \delta_{i, R}\right)\right) .
$$

When $\delta_{i, R}=\frac{\alpha_{i} \beta_{i}}{4 C_{1}\left(\alpha_{i}+\beta_{i}\right)}$ this is immediate from Theorem 1.4;

$$
M_{1} \subseteq M^{\prime} \subseteq \mathbb{R}^{3} \backslash\left(\bigcup_{i} B_{\delta}\left(p_{i}, \frac{\alpha_{i} \beta_{i}}{4 C_{1}\left(\alpha_{i}+\beta_{i}\right)}\right)\right)
$$

In the case of a long cylindrical end, when $\delta_{i, R}=\left(\alpha_{i}+\beta_{i}\right) \exp \left(-R /\left(\alpha_{i}+\beta_{i}\right)\right)$, we obtain (4.14) by proving that

$$
d_{g}\left(0, \partial B_{\delta}\left(p_{i}, \delta_{i, R}\right)\right)>R .
$$

Using $\alpha_{i}+\beta_{i}<m<\sigma \leq\left|p_{i}\right|$ and the fact that

$$
g>\left(1+\frac{\alpha_{i}}{\left|x-p_{i}\right|}\right)^{2}\left(1+\frac{\beta_{i}}{\left|x-p_{i}\right|}\right)^{2} \delta
$$

we can compute:

$$
\begin{aligned}
d_{g}\left(0, \partial B_{\delta}\left(p_{i}, \delta_{i, R}\right)\right) & >d_{g}\left(\partial B_{\delta}\left(p_{i}, \alpha_{i}+\beta_{i}\right), \partial B_{\delta}\left(p_{i}, \delta_{i, R}\right)\right) \\
& =\int_{t=0}^{1} g\left(\gamma^{\prime}(t), \gamma^{\prime}(t)\right)^{1 / 2} d t
\end{aligned}
$$
where $\gamma$ is a minimal geodesic

$$
\begin{aligned}
& =\int_{t=0}^{1}\left(1+\frac{\alpha_{i}}{\left|\gamma(t)-p_{i}\right|}\right)\left(1+\frac{\beta_{i}}{\left|\gamma(t)-p_{i}\right|}\right)\left|\gamma^{\prime}(t)\right| d t \\
& \geq \int_{t=0}^{1}\left(1+\frac{\alpha_{i}}{r}\right)\left(1+\frac{\beta_{i}}{r}\right) \frac{d}{d t}(r(\gamma(t))) d t
\end{aligned}
$$$$
\geq \int_{\delta_{i, R}}^{\alpha_{i}+\beta_{i}}\left(1+\frac{\alpha_{i}}{r}\right)\left(1+\frac{\beta_{i}}{r}\right) d r
$$$$
>\int_{\delta_{i, R}}^{\alpha_{i}+\beta_{i}} \frac{\alpha_{i}+\beta_{i}}{r} d r>\left(\alpha_{i}+\beta_{i}\right) \ln \left(\frac{\alpha_{i}+\beta_{i}}{\delta_{i, R}}\right)=R .
$$

This gives us (4.16) which implies (4.14), and we are done. 


\subsection{Proximity of $g$ to $\delta$}

We continue by identifying a region of $M^{\prime}$ where $g$ is close to $\delta$ in the sense of (4.1). We note that the results of this subsection are independent of the parameter $R$.

Lemma 4.5. Let $\varepsilon>0$ and assume that $m<\varepsilon \cdot \frac{\sigma}{16}$. Then on

$$
\mathbb{R}^{3} \backslash\left(\bigcup_{i=1}^{n} B_{\delta}\left(p_{i}, \frac{8}{\varepsilon}\left(\alpha_{i}+\beta_{i}\right)\right)\right) .
$$

we have

$$
\delta \leq g \leq(1+\varepsilon)^{2} \delta .
$$

Proof. It suffices to prove that

$$
1+\sum_{i} \frac{\alpha_{i}}{\left|x-p_{i}\right|}<1+\varepsilon / 4 \text { and } 1+\sum_{i} \frac{\beta_{i}}{\left|x-p_{i}\right|}<1+\frac{\varepsilon}{4}
$$

Our hypothesis on $m$ gives us

$$
\frac{8}{\varepsilon}\left(\alpha_{i}+\beta_{i}\right)<\frac{8 m}{\varepsilon}<\frac{\sigma}{2} .
$$

Suppose $x \notin \bigcup_{i} B_{\delta}\left(p_{i}, \frac{8}{\varepsilon}\left(\alpha_{i}+\beta_{i}\right)\right)$. In the case when $x \notin \bigcup_{i} B_{\delta}\left(p_{i}, \sigma / 2\right)$ we have

$$
\sum_{i} \frac{\alpha_{i}}{\left|x-p_{i}\right|}<2 \sum_{i} \frac{\alpha_{i}}{\sigma}<2 \frac{m}{\sigma}<\frac{\varepsilon}{8}
$$

and an analogous inequality with $\beta_{i}$. On the other hand, if $\left|x-p_{j}\right|<\sigma / 2$ for some (and hence exactly one) $j$ then

$$
\sum_{i} \frac{\alpha_{i}}{\left|x-p_{i}\right|}<\frac{\varepsilon \alpha_{j}}{8\left(\alpha_{j}+\beta_{j}\right)}+2 \sum \frac{\alpha_{i}}{\sigma}<\frac{\varepsilon}{8}\left(\frac{\alpha_{j}}{\alpha_{j}+\beta_{j}}+1\right)<\frac{\varepsilon}{4} .
$$

(An analogous inequality can be proven for $\beta$ 's as well.)

For a fixed $\varepsilon>0$, Brill-Lindquist geometrostatic manifolds whose ADM mass satisfies $m<\sigma /\left(20 C_{1}\right)$ and $m<\varepsilon \cdot \frac{\sigma}{16}$, and lengths $\gamma_{i}$ from (3.154) we define

$$
\gamma_{i, \varepsilon}=\max \left\{\frac{8}{\varepsilon}\left(\alpha_{i}+\beta_{i}\right), \gamma_{i}\right\} .
$$

The purpose of introducing $\gamma_{i, \varepsilon}$ is in marking the portion of $M^{\prime}$

$$
\mathbb{R}^{3} \backslash\left(\bigcup_{i=1}^{n} B_{\delta}\left(p_{i}, \gamma_{i, \varepsilon}\right)\right) \subseteq M^{\prime}
$$

on which the metric $g$ is suitably close to the Euclidean metric $\delta$ :

$$
\delta \leq g \leq(1+\varepsilon)^{2} \delta .
$$

We now record several estimates involving $\gamma_{i, \varepsilon}$ which are needed later. 
Lemma 4.6. Let $0<\varepsilon<\varepsilon_{0}:=\sqrt{\frac{2}{\pi C_{1}^{2}}}$. We have

(1) $\gamma_{i, \varepsilon} \leq 8 m / \varepsilon$ for all $1 \leq i \leq n$;

(2) $\sum \gamma_{i, \varepsilon}^{2}<96 m^{2} / \varepsilon^{2}$;

(3) $\sum \gamma_{i, \varepsilon}^{3}<768 m^{3} / \varepsilon^{3}$;

Proof. Since $\alpha_{i}+\beta_{i}<\sum\left(\alpha_{i}+\beta_{i}\right)=m$, the first claim follows from 3.155):

$$
\gamma_{i, \varepsilon} \leq 8 m \max \left\{\frac{1}{\varepsilon}, C_{1}\right\}=\frac{8 m}{\varepsilon},
$$

and so does the second claim,

$$
\begin{aligned}
\sum \gamma_{i, \varepsilon}^{2} & \leq \frac{64}{\varepsilon^{2}} \sum m\left(\alpha_{i}+\beta_{i}\right)+C_{1}^{2} \sum \operatorname{Area}_{g}\left(\Sigma_{i}\right) \\
& \leq \frac{64 m^{2}}{\varepsilon^{2}}+16 \pi C_{1}^{2} m^{2}<96 \frac{m^{2}}{\varepsilon^{2}} .
\end{aligned}
$$

The third estimate is immediate from the first two.

\subsection{Estimating lengths}

In this subsection we estimate the length parameter $\lambda$ of (4.4).

Lemma 4.7. Assume that

$$
m<\varepsilon \cdot \frac{\sigma}{32} \text { and } m<R \varepsilon^{3} \text { where } 0<\varepsilon<\varepsilon_{0}:=\sqrt{\frac{2}{\pi C_{1}^{2}}} .
$$

Furthermore, let

$$
W^{\prime}=B_{\delta}(0, R) \backslash\left(\bigcup_{i} B_{\delta}\left(p_{i}, \gamma_{i, \varepsilon}\right)\right)
$$

Then $W^{\prime} \subseteq M^{\prime}$ and the parameter

$$
\lambda:=\sup _{x, y \in W^{\prime}}\left|d_{\left(M^{\prime}, g\right)}(x, y)-d_{\left(\mathbb{R}^{3}, \delta\right)}(x, y)\right|
$$

satisfies

$$
\lambda<\lambda_{R, \varepsilon}:=24 R \varepsilon .
$$

In particular, $\lambda$ scales like distance and converges to 0 for fixed $R$ as $\varepsilon$ to 0 .

Note that this $\lambda$ will be useful for estimating the parameter (4.4) for any set $W \subseteq W^{\prime}$ as well.

Proof. It follows from (4.36) that $\sigma>20 m C_{1}$. Consequently, (4.32) applies and we have $W^{\prime} \subseteq M^{\prime}$. Now let $x, y \in W^{\prime}$. These two points can be joined by a path $\varphi$ in $M^{\prime}$ consisting of portions of the Euclidean line segment $x y$ and interrupted by several at most semi-circular arcs along the spheres of Euclidean radius $\gamma_{i, \varepsilon}$ (for 
varying $i$ ) by Proposition 3.19 and definitions (3.154) and (4.31). The centers of these spheres project onto points on the line segment $x y$ which are at least

$$
\sqrt{\sigma^{2}-\max _{i}\left\{2 \gamma_{i, \varepsilon}\right\}^{2}} \geq \sqrt{\sigma^{2}-\left(\frac{16 m}{\varepsilon}\right)^{2}}>\sqrt{\sigma^{2}-\left(\frac{\sigma}{2}\right)^{2}}>\frac{\sigma}{2}
$$

away from each other; consult the diagram below for details.

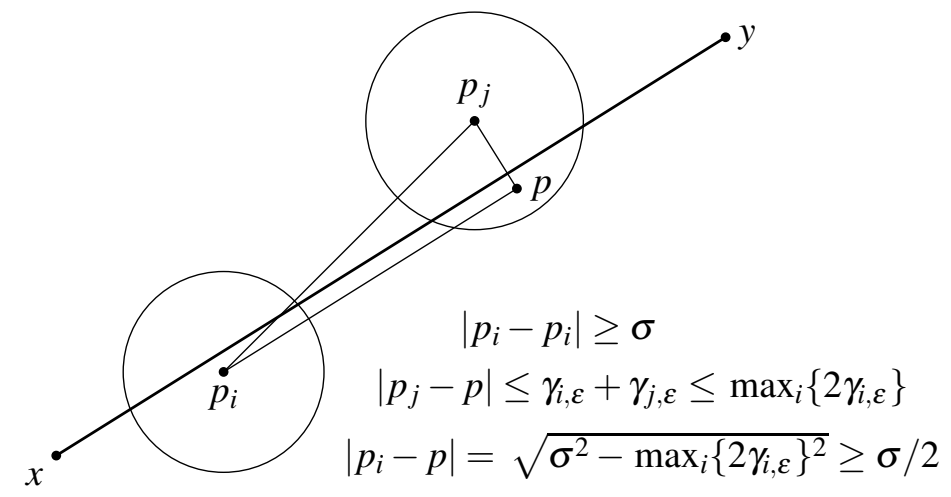

FIGURE 4.1. Illustration for the proof of Lemma 4.7

It follows from $|x-y|<2 R$ that there can be no more than $\frac{4 R}{\sigma}$ arcs on the path $\varphi$. In particular, the length of $\varphi$ measured with respect to Euclidean metric satisfies

$$
d_{\left(M^{\prime}, \delta\right)}(x, y) \leq L_{\delta}(\varphi) \leq|x-y|+\pi \sum \gamma_{i, \varepsilon}
$$

where the summation in the last line goes over at most $\frac{4 R}{\sigma}$ elements. By CauchySchwarz inequality we see that the latter sum satisfies

$$
\left(\sum \gamma_{i, \varepsilon}\right)^{2} \leq \frac{4 R}{\sigma} \sum \gamma_{i, \varepsilon}^{2}
$$

It follows from Lemma 4.6 and the assumption $m<\varepsilon \cdot \frac{\sigma}{32}$ that

$$
\sum \gamma_{i, \varepsilon}^{2}<96 m^{2} / \varepsilon^{2}<3 \sigma m / \varepsilon
$$

Overall, we see that the summation term in (4.41) can be bounded by

$$
\left(\sum \gamma_{i, \varepsilon}\right)^{2} \leq \frac{4 R}{\sigma} \cdot \frac{3 \sigma m}{\varepsilon} \leq \frac{12 m R}{\varepsilon}
$$

Combining this with (4.41) we have

$$
d_{\left(M^{\prime}, \delta\right)}(x, y) \leq|x-y|+\pi \sum \gamma_{i, \varepsilon}<d_{\left(\mathbb{R}^{3}, \delta\right)}(x, y)+\pi \sqrt{\frac{12 m R}{\varepsilon}} .
$$

By Lemma 4.5 we have

$$
d_{\left(\mathbb{R}^{3}, \delta\right)}(x, y) \leq d_{\left(M^{\prime}, \delta\right)}(x, y) \leq d_{\left(M^{\prime}, g\right)}(x, y) \leq(1+\varepsilon) d_{\left(M^{\prime}, \delta\right)}(x, y) .
$$


Since $m<R \varepsilon^{3}$ the estimate (4.45) implies

$$
d_{\left(\mathbb{R}^{3}, \delta\right)}(x, y) \leq d_{\left(M^{\prime}, g\right)}(x, y) \leq(1+\varepsilon)\left(d_{\left(\mathbb{R}^{3}, \delta\right)}(x, y)+\pi R \varepsilon \sqrt{12}\right) .
$$

It follows from $d_{\left(\mathbb{R}^{3}, \delta\right)}(x, y)=|x-y|<2 R$ that

$$
0 \leq d_{\left(M^{\prime}, g\right)}(x, y)-d_{\left(\mathbb{R}^{3}, \delta\right)}(x, y) \leq R \varepsilon(2+(1+\varepsilon) \pi \sqrt{12}) .
$$

The claim (4.39) is now immediate from $2+(1+\varepsilon) \pi \sqrt{12}<2+4 \pi \sqrt{3}<24$.

Remark 4.8. Note that in Lemma4.7 our estimates do not depend upon the number of points $p_{i} \in P$ nor on the number pf minimal surfaces $\Sigma_{i}$.

\subsection{Introducing $W$}

In order to apply Theorem 4.1 we need a pair of diffeomorphic subregions $W_{1}$ and $W_{2}$. We are able to simplify the situation slightly by choosing a single $W \subseteq \mathbb{R}^{3}$ which can be viewed as both a subset of $M_{1}$ and $M_{2}$ of (4.6)-4.7). Our $W^{\prime}$ defined in (4.37) may not be a subset of both these manifolds.

Define

$$
W=B_{\delta}(0, R-\lambda) \backslash\left(\bigcup_{i} B_{\delta}\left(p_{i}, \gamma_{i, \varepsilon}\right)\right),
$$

where $\lambda$ is the parameter estimated in Lemma 4.7 and $\gamma_{i, \varepsilon}$ is defined in (4.31). Clearly, $W=W^{\prime} \cap B_{\delta}(0, R-\lambda) \subseteq W^{\prime}$ of Lemma 4.7. See Figure 4.2 for two different scenarios as to how $W \subseteq M_{1} \subseteq M^{\prime}$ depending on which whether the $i^{t h}$ minimal surface $\Sigma_{i}$ is located outside or inside of $B_{\delta}\left(p_{i}, \delta_{i, R}\right)$.
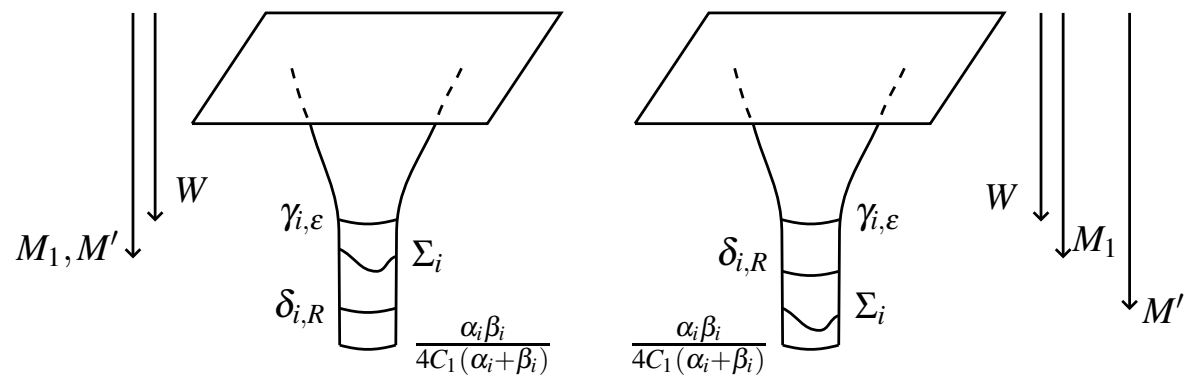

FIGURE 4.2. Two scenarios.

The following records the properties of $W$ needed in order to apply Theorem 4.1

Corollary 4.9. Assume that

$$
m<\varepsilon \cdot \frac{\sigma}{32} \text { and } m<R \varepsilon^{3} \text { where } 0<\varepsilon<\varepsilon_{0}:=\sqrt{\frac{2}{\pi C_{1}^{2}}} .
$$


I We have

$$
W \subseteq M_{1} \cap M_{2},
$$

where $M_{1}$ is as in (4.6) and $M_{2}$ is as in (4.7).

II The following estimate holds over $W$ :

$$
\delta \leq g \leq(1+\varepsilon)^{2} \delta
$$

III The parameter

$$
\lambda:=\sup _{x, y \in W}\left|d_{\left(M^{\prime}, g\right)}(x, y)-d_{\left(\mathbb{R}^{3}, \delta\right)}(x, y)\right|
$$

satisfies

$$
\lambda<\lambda_{R, \varepsilon}=24 R \varepsilon
$$

IV We have

$$
M_{1} \backslash W \subseteq\left(B_{\delta}(0, R) \backslash B_{\delta}(0, R-\lambda)\right) \cup \bigcup_{i}\left(B_{\delta}\left(p_{i}, \gamma_{i, \varepsilon}\right) \backslash B_{\delta}\left(p_{i}, \delta_{i, R}\right)\right),
$$

where clearly $B_{\delta}\left(p_{i}, \gamma_{i, \varepsilon}\right) \backslash B_{\delta}\left(p_{i}, \delta_{i, R}\right)=\emptyset$ when $\gamma_{i, \varepsilon} \leq \delta_{i, R}$ of (4.11).

$\mathrm{V}$ We have

$$
M_{2} \backslash W=\left(B_{\delta}(0, R) \backslash B_{\delta}(0, R-\lambda)\right) \cup \bigcup_{i} B_{\delta}\left(p_{i}, \gamma_{i, \varepsilon}\right) .
$$

VI Suppose that

$$
\rho(P) \cap(R-32 R \varepsilon, R+32 R \varepsilon)=\emptyset
$$

where $\rho(x)=|x|$ and $P=\left\{p_{1}, \ldots, p_{N}\right\}$. Then the unions in parts (IV) and (V) of this corollary are disjoint.

Proof. Let $x \in W$. Since by definition $W \subseteq W^{\prime}$ and since $W^{\prime} \subseteq M^{\prime}$ by Lemma 4.7 we have that $W \subseteq M^{\prime}$. Furthermore, we see from

$$
d_{\left(M^{\prime}, g\right)}(0, x)<|x|+\lambda<R
$$

that $x \in B_{g}(0, R) \cap M^{\prime}=M_{1}$. Observing that $x \in B_{\delta}(0, R-\lambda) \subset M_{2}$ completes the proof of par $\Pi$. Parts $\Pi$ and $\Pi$ are now immediate from Lemmas 4.7 and 4.5 , respectively. Note that hypotheses (4.50) imply $m<\sigma\left(20 C_{1}\right)$ so that Lemma 4.4 applies. Consequently, we have parts IV and $\mathrm{V}]$ of our corollary. It remains to assume (4.57) and argue that the unions in parts IV] and $\mathrm{V}$ are disjoint:

$$
B_{\delta}(0, R) \backslash B_{\delta}(0, R-\lambda) \subset \mathbb{R}^{3} \backslash \bigcup_{i} B_{\delta}\left(p_{i}, \gamma_{i, \varepsilon}\right)
$$

Assumption (4.57), and Lemma 4.6, imply

$$
|| p_{i}|-R| \geq 32 R \varepsilon>\lambda_{R, \varepsilon}+8 R \varepsilon^{2}>\lambda+\frac{8 m}{\varepsilon} \geq \lambda+\gamma_{i, \varepsilon}>\gamma_{i, \varepsilon} .
$$

for all $1 \leq i \leq n$. In particular, we have

$$
\left|p_{i}\right|<R-\lambda-\gamma_{i, \varepsilon} \text { or }\left|p_{i}\right|>R+\gamma_{i, \varepsilon}
$$


for all $1 \leq i \leq n$ and the inclusion in 4.59) is now immediate.

\subsection{Estimating Volumes}

To complete the estimation of the intrinsic flat distance between $\left(M_{1}, g\right)$ and $\left(M_{2}, \delta\right)$ we need estimates on volumes and areas in the hypothesis of Theorem 4.1 . This is done in Lemma 4.10 and Lemma4.11.

Lemma 4.10. Let $\varepsilon_{0}:=\sqrt{\frac{2}{\pi C_{1}^{2}}} \in(0,1)$. There exists a universal constant $C^{\prime}>0$ such that for all $R>0$, all $\varepsilon \in\left(0, \varepsilon_{0}\right)$, and all geometrostatic manifolds $(M, g)$ with

$$
m_{A D M}\left(M^{\prime}\right)=m<R \varepsilon^{3}, \quad m<\varepsilon \cdot \frac{\sigma}{32}
$$

the region $W$ of (4.49) and Corollary 4.9 satisfies

$$
\begin{aligned}
& \operatorname{Vol}_{\delta}(W) \leq \operatorname{Vol}_{g}(W) \leq C^{\prime} R^{3} \\
& \operatorname{Vol}_{\delta}(\partial W) \leq \operatorname{Vol}_{g}(\partial W) \leq C^{\prime} R^{2}
\end{aligned}
$$

Proof. Fix $\varepsilon$ with $0<\varepsilon<\varepsilon_{0}$. The first estimate follows from the fact that

$$
\begin{aligned}
\operatorname{Vol}_{g}(W) & \leq(1+\varepsilon)^{3} \operatorname{Vol}_{\delta}(W) \text { by Lemma } 4.5 \\
& \leq(1+\varepsilon)^{3} \operatorname{Vol}_{\delta}\left(B_{\delta}(0, R-\lambda)\right) \text { by } W \subset B_{\delta}(0, R-\lambda), \\
& \leq \frac{4 \pi}{3}\left(1+\varepsilon_{0}\right)^{3}(R-\lambda)^{3} \leq C^{\prime} R^{3}
\end{aligned}
$$

for some universal constant $C^{\prime}>0$. To prove the second estimate we have:

$$
\begin{aligned}
\operatorname{Vol}_{g}(\partial W) & \leq(1+\varepsilon)^{2} \operatorname{Vol}_{\delta}(\partial W) \text { by Lemma 4.5, } \\
& \leq 4 \pi(1+\varepsilon)^{2}\left(R^{2}+\sum \gamma_{i, \varepsilon}^{2}\right) \text { by 4.49), } \\
& \leq 4 \pi(1+\varepsilon)^{2}\left(R^{2}+96 m^{2} / \varepsilon^{2}\right) \text { by Lemma 4.6. } \\
& \leq 4 \pi\left(1+\varepsilon_{0}\right)^{2}\left(R^{2}+96 R^{2} \varepsilon_{0}^{4}\right) \\
& \leq C^{\prime} R^{2}
\end{aligned}
$$

for some universal constant $C^{\prime}>0$.

Lemma 4.11. Let $\varepsilon_{0}:=\sqrt{\frac{2}{\pi C_{1}^{2}}} \in(0,1)$. There exists a universal constant $C^{\prime \prime}>0$ such that for all $R>0$, all $\varepsilon \in\left(0, \varepsilon_{0}\right)$, and all geometrostatic manifolds $(M, g)$ with (4.69) $m_{A D M}\left(M^{\prime}\right)=m<R \varepsilon^{3}, \quad m<\varepsilon \cdot \frac{\sigma}{32}, \quad \rho(P) \cap(R-32 R \varepsilon, R+32 R \varepsilon)=\emptyset$, the regions $M_{1}$ of (4.6), $M_{2}$ of (4.7), and $W$ of (4.49) and Corollary 4.9 satisfy

$$
\begin{aligned}
& \operatorname{Vol}_{g}\left(M_{1} \backslash W\right) \leq C^{\prime \prime} R^{3} \varepsilon, \\
& \operatorname{Vol}_{\delta}\left(M_{2} \backslash W\right) \leq C^{\prime \prime} R^{3} \varepsilon .
\end{aligned}
$$


Proof. Fix $\varepsilon$ with $0<\varepsilon<\varepsilon_{0}$. We start by proving (4.71), as it is easier to establish. Lemma 4.6 and parts (III) and (V) of Corollary 4.9 imply that

$$
\begin{aligned}
\operatorname{Vol}_{\delta}\left(M_{2} \backslash W\right) & =\operatorname{Vol}_{\delta}\left(B_{\delta}(0, R) \backslash B_{\delta}(0, R-\lambda)\right)+\sum_{i} \operatorname{Vol}_{\delta}\left(B_{\delta}\left(p_{i}, \gamma_{i, \varepsilon}\right)\right) \\
& =\frac{4}{3} \pi R^{3}-\frac{4}{3} \pi(R-\lambda)^{3}+\sum_{i} \frac{4}{3} \pi\left(\gamma_{i, \varepsilon}\right)^{3} \\
& \leq \frac{4 \pi}{3}\left(3 R^{2} \lambda+\lambda^{3}+768 m^{3} / \varepsilon^{3}\right) \\
& \leq \frac{4 \pi}{3}\left(3 R^{2}(24 R \varepsilon)+(24 R \varepsilon)^{3}+768 R^{3} \varepsilon^{6}\right) \\
& \leq C^{\prime \prime} R^{3} \varepsilon
\end{aligned}
$$

for some universal constant $C^{\prime \prime}$.

The inequality (4.70) is far more difficult to prove. By part (IV) of Proposition 4.9 (see Figure 4.2) we have

$$
\operatorname{Vol}_{g}\left(M_{1} \backslash W\right) \leq \operatorname{Vol}_{g}\left(B_{\delta}(0, R) \backslash B_{\delta}(0, R-\lambda)\right)+\sum_{i} V_{i}
$$

where

$$
V_{i}:=\operatorname{Vol}_{g}\left(B_{\delta}\left(p_{i}, \gamma_{i, \varepsilon}\right) \backslash B_{\delta}\left(p_{i}, \delta_{i, R}\right)\right) .
$$

We see from part (VI) of Corollary 4.9 that (4.32) and (4.33) apply, giving us (4.79) $\operatorname{Vol}_{g}\left(B_{\delta}(0, R) \backslash B_{\delta}(0, R-\lambda)\right) \leq(1+\varepsilon)^{3} \operatorname{Vol}_{\delta}\left(B_{\delta}(0, R) \backslash B_{\delta}(0, R-\lambda)\right)$

$$
\begin{aligned}
& \leq(1+\varepsilon)^{3}\left(\frac{4}{3} \pi R^{3}-\frac{4}{3} \pi(R-\lambda)^{3}\right) \\
& \leq \frac{4}{3} \pi\left(1+\varepsilon_{0}\right)^{3}\left(3 R^{2} \lambda+\lambda^{3}\right) .
\end{aligned}
$$

Combining this with (4.77) and part (III) of Corollary 4.9 we have

$$
\operatorname{Vol}_{g}\left(M_{1} \backslash W\right) \leq \frac{4}{3} \pi\left(1+\varepsilon_{0}\right)^{3}\left(3 R^{2}(24 R \varepsilon)+(24 R \varepsilon)^{3}\right)+\sum_{i} V_{i} .
$$

Next we estimate each term, $V_{i}$, in the sum. Let $x \in B_{\delta}\left(p_{i}, \gamma_{i, \varepsilon}\right) \backslash B_{\delta}\left(p_{i}, \delta_{i, R}\right)$ and let $j \neq i$. The definition of $\sigma$ in (1.7), Lemma 4.6 and our hypothesis (4.69) imply

$$
\left|x-p_{j}\right| \geq\left|p_{i}-p_{j}\right|-\left|x-p_{i}\right|>\sigma-\gamma_{i, \varepsilon} \geq \sigma-(8 m / \varepsilon)>24 m / \varepsilon .
$$

Combining this with $\sum_{j}\left(\alpha_{j}+\beta_{j}\right) \leq m$ in (1.4) we have

$$
1+\sum_{j \neq i} \frac{\alpha_{j}}{\left|x-p_{j}\right|}<1+\frac{\varepsilon}{24}<2 \quad \text { and } \quad 1+\sum_{j \neq i} \frac{\beta_{j}}{\left|x-p_{j}\right|}<1+\frac{\varepsilon}{24}<2 .
$$

In particular, it follows that

$$
V_{i} \leq \int_{\delta_{i, R}}^{\gamma_{i, \varepsilon}}\left(2+\frac{\alpha_{i}}{r}\right)^{3}\left(2+\frac{\beta_{i}}{r}\right)^{3} \pi r^{2} d r
$$


After expanding the integrand in terms of powers of $r$ and integrating individual terms we obtain:

$$
\begin{aligned}
V_{i} \leq & (64 / 3) \pi \gamma_{i, \varepsilon}^{3}+48 \pi\left(\alpha_{i}+\beta_{i}\right) \gamma_{i, \varepsilon}^{2} \\
& +48 \pi\left(\alpha_{i} \beta_{i}+\left(\alpha_{i}+\beta_{i}\right)^{2}\right) \gamma_{i, \varepsilon} \\
& -8 \pi\left(\alpha_{i}+\beta_{i}\right)\left(\left(\alpha_{i}+\beta_{i}\right)^{2}+6 \alpha_{i} \beta_{i}\right) \ln \left(\delta_{i, R} / \gamma_{i, \varepsilon}\right) \\
& +12 \pi\left(\alpha_{i} \beta_{i}+\left(\alpha_{i}+\beta_{i}\right)^{2}\right)\left(\alpha_{i} \beta_{i}\right) / \delta_{i, R} \\
& +6 \pi\left(\alpha_{i}+\beta_{i}\right)\left(\left(\alpha_{i} \beta_{i}\right) / \delta_{i, R}\right)^{2}+\pi\left(\left(\alpha_{i} \beta_{i}\right) /\left(\delta_{i, R}\right)\right)^{3}
\end{aligned}
$$

By the definition of $\delta_{i, R}$ in 4.11) and Lemma 4.6, we have

$$
\frac{\delta_{i, R}}{\gamma_{i, \varepsilon}} \geq \frac{\varepsilon\left(\alpha_{i}+\beta_{i}\right) \exp \left(-R /\left(\alpha_{i}+\beta_{i}\right)\right)}{8 m} .
$$

Thus

$$
-\ln \left(\frac{\delta_{i, R}}{\gamma_{i, \varepsilon}}\right) \leq-\ln \left(\frac{\varepsilon\left(\alpha_{i}+\beta_{i}\right)}{8 m}\right)+\frac{R}{\left(\alpha_{i}+\beta_{i}\right)}
$$

Since $\sup _{x>0}(-x \ln (x))=e^{-1}<1 / 2$ we have $-\ln (x)<1 /(2 x)$ for positive $x$. So

$$
-\ln \left(\frac{\varepsilon\left(\alpha_{i}+\beta_{i}\right)}{8 m}\right) \leq \frac{4 m}{\varepsilon\left(\alpha_{i}+\beta_{i}\right)} .
$$

Combining this with the above and multiplying by $\left(\alpha_{i}+\beta_{i}\right)$ we get,

$$
-\left(\alpha_{i}+\beta_{i}\right) \ln \left(\frac{\delta_{i, R}}{\gamma_{i, \varepsilon}}\right) \leq \frac{4 m}{\varepsilon}+R .
$$

Furthermore by 4.11)

$$
\frac{\alpha_{i} \beta_{i}}{\delta_{i, R}} \leq 4 C_{1}\left(\alpha_{i}+\beta_{i}\right)
$$

Together with estimates such as $4 \alpha_{i} \beta_{i} \leq\left(\alpha_{i}+\beta_{i}\right)^{2}$ we have

$$
\begin{aligned}
\sum_{i} V_{i} \leq & (64 / 3) \pi \sum_{i} \gamma_{i, \varepsilon}^{3}+48 \pi \sum_{i}\left(\alpha_{i}+\beta_{i}\right) \gamma_{i, \varepsilon}^{2} \\
& +48 \pi \sum_{i}\left[\left(\alpha_{i}+\beta_{i}\right)^{2} / 4+\left(\alpha_{i}+\beta_{i}\right)^{2}\right] \gamma_{i, \varepsilon} \\
& +8 \pi \sum_{i}\left(\left(\alpha_{i}+\beta_{i}\right)^{2}+6\left(\alpha_{i}+\beta_{i}\right)^{2} / 4\right)\left(\frac{4 m}{\varepsilon}+R\right) \\
& +12 \pi \sum_{i}\left(\left(\alpha_{i}+\beta_{i}\right)^{2} / 4+\left(\alpha_{i}+\beta_{i}\right)^{2}\right) 4 C_{1}\left(\alpha_{i}+\beta_{i}\right) \\
& +6 \pi \sum_{i}\left(\alpha_{i}+\beta_{i}\right)\left(4 C_{1}\left(\alpha_{i}+\beta_{i}\right)\right)^{2}+\pi \sum_{i}\left(4 C_{1}\left(\alpha_{i}+\beta_{i}\right)\right)^{3}
\end{aligned}
$$


By Lemma 4.6 we have
(4.101) $\quad \sum_{i} \gamma_{i, \varepsilon}^{3}$
$<768 m^{3} / \varepsilon^{3}$
(4.102)

$$
\sum_{i}\left(\alpha_{i}+\beta_{i}\right) \gamma_{i, \varepsilon}^{2}<96 m^{3} / \varepsilon^{2}
$$

$$
\begin{aligned}
\sum_{i}\left(\alpha_{i}+\beta_{i}\right)^{2} \gamma_{i, \varepsilon} & \leq\left(\sum_{i}\left(\alpha_{i}+\beta_{i}\right)^{4}\right)^{1 / 2}\left(\sum_{i} \gamma_{i, \varepsilon}^{2}\right)^{1 / 2} \\
& <\left(\sum_{i}\left(\alpha_{i}+\beta_{i}\right)\right)^{2} \sqrt{96 m^{2} / \varepsilon^{2}}<10 m^{3} / \varepsilon
\end{aligned}
$$

with the last set of estimates holding due to

$$
\sum_{i}\left(\alpha_{i}+\beta_{i}\right)^{4}<\left(\sum_{i}\left(\alpha_{i}+\beta_{i}\right)\right)^{4} \leq m^{4} .
$$

Likewise, $\sum_{i}\left(\alpha_{i}+\beta_{i}\right)^{3}<m^{3}$ and thus

$$
\begin{aligned}
\sum_{i} V_{i} \leq & (64 / 3) \pi \cdot 768 m^{3} / \varepsilon^{3}+48 \pi \cdot 96 m^{3} / \varepsilon^{2}+60 \pi \cdot 10 m^{3} / \varepsilon \\
& +20 \pi m^{2}(4 m / \varepsilon+R)+60 \pi C_{1} m^{3}+96 \pi C_{1}^{2} m^{3}+64 \pi C_{1}^{3} m^{3} \\
\leq & (64 / 3) \pi \cdot 768 R^{3} \varepsilon^{6}+48 \pi \cdot 96 R^{3} \varepsilon^{7}+60 \pi \cdot 10 R^{3} \varepsilon^{8} \\
& +20 \pi R^{3} \varepsilon^{6}\left(4 \varepsilon^{2}+1\right)+60 \pi C_{1} R^{3} \varepsilon^{9}+96 \pi C_{1}^{2} R^{3} \varepsilon^{9}+64 \pi C_{1}^{3} R^{3} \varepsilon^{9} \\
\leq & C^{\prime \prime} R^{3} \varepsilon^{6}
\end{aligned}
$$

for some universal constant $C^{\prime \prime}$. Combining this with 4.82) we finally have our first inequality.

\subsection{The proof of Proposition 4.2}

We now prove Proposition 4.2 bounding the intrinsic flat distance between

$$
M_{1}=\left\{x \in M^{\prime} \mid d_{\left(M^{\prime}, g\right)}(0, x)<R\right\}=B_{g}(0, R) \subseteq M^{\prime}
$$

endowed with the distance $d_{\left(M^{\prime}, g\right)}$ and

$$
M_{2}=\left\{x \in \mathbb{R}^{3}|| x \mid<R\right\}=B_{\delta}(0, R)
$$

endowed with the distance $d_{\left(\mathbb{R}^{3}, d_{\delta}\right)}(x, y)=|x-y|$. Note that the following estimate on the diameters of these regions

$$
\max \left\{\operatorname{diam}_{g}\left(M_{1}\right), \operatorname{diam}_{\delta}\left(M_{2}\right)\right\} \leq D=2 R .
$$

Proof of Proposition 4.2 We prove this proposition using the method of Lakzian and the first author to estimate the intrinsic flat distance (cf. Theorem 4.1):

$$
\begin{aligned}
d_{\mathscr{F}}\left(M_{1}, M_{2}\right) \leq & (2 \bar{h}+a)\left(\operatorname{Vol}_{g}(W)+\operatorname{Vol}_{\delta}(W)+\operatorname{Vol}_{g}(\partial W)+\operatorname{Vol}_{\delta}(\partial W)\right) \\
& +\operatorname{Vol}_{g}\left(M_{1} \backslash W\right)+\operatorname{Vol}_{\delta}\left(M_{2} \backslash W\right)
\end{aligned}
$$


taking $W$ as defined in (4.49) and addressed in detail in Corollary 4.9

Let $\varepsilon_{0}:=\sqrt{\frac{2}{\pi C_{1}^{2}}} \in(0,1)$ and let $0<\varepsilon<\varepsilon_{0}$. Define $a$ as in (4.3):

$$
a=\frac{\arccos \left[(1+\varepsilon)^{-1}\right]}{\pi} \cdot D=\frac{\arccos \left[(1+\varepsilon)^{-1}\right]}{\pi} \cdot 2 R .
$$

It follows from the L'Hôpital's Rule that

$$
\varepsilon \mapsto \varepsilon^{-1 / 2} \cdot \arccos \left((1+\varepsilon)^{-1}\right)
$$

is a positive bounded function of $\varepsilon \in[0, \infty)$. Thus there is a universal constant $C_{a}$ such that

$$
0<a \leq C_{a} R \sqrt{\varepsilon}
$$

In addition, by part (III) of Corollary 4.9 we have that $\bar{h}$ of (4.5) satisfies

$$
\begin{aligned}
\bar{h} & =\max \left\{\sqrt{2 \lambda D}, D \sqrt{\varepsilon^{2}+2 \varepsilon}\right\} \\
& \leq \max \{\sqrt{2(24 R \varepsilon) 2 R}, 2 R \sqrt{3 \varepsilon}\} \leq 10 R \sqrt{\varepsilon} .
\end{aligned}
$$

Overall, we see that

$$
0<2 \bar{h}+a \leq C_{2} R \sqrt{\varepsilon}
$$

for some universal constant $C_{2}$. Substituting our bound on $2 \bar{h}+a$ into (4.114) along with the volume estimates from Lemmas 4.10 and 4.11 we have,

$$
d_{\mathscr{F}}\left(M_{1}, M_{2}\right) \leq C_{2} R \sqrt{\varepsilon} \cdot\left(2 C^{\prime} R^{3}+2 C^{\prime} R^{2}\right)+2 C^{\prime \prime} R^{3} \varepsilon
$$

which in turn implies (4.9). Similarly, using $D=2 R$ we have

$$
d_{D \mathscr{F}}\left(M_{1}, M_{2}\right) \leq \frac{1}{2 R} \cdot C_{2} R \sqrt{\varepsilon} \cdot\left(2 C^{\prime} R^{3}+2(2 R) C^{\prime} R^{2}\right)+2 C^{\prime \prime} R^{3} \varepsilon,
$$

which implies 4.10).

\subsection{The proof of Theorem 1.5}

Proof. By assumption there is some $R_{0} \geq 0$ such that for almost every $R>R_{0}, R$ is not an accumulation point of $\rho\left(\cup_{k} P_{k}\right)$. Fix one such value of $R$. We show that for all $\bar{\varepsilon}>0$ there is $K=K(\bar{\varepsilon}) \in \mathbb{N}$ such that

$$
d_{\mathscr{F}}\left(B_{g_{k}}(0, R), B_{\delta}(0, R)\right)<\bar{\varepsilon} \text { and } d_{D \mathscr{F}}\left(B_{g_{k}}(0, R), B_{\delta}(0, R)\right)<\bar{\varepsilon}
$$

for all $k \geq K$.

For our fixed value of $R$ there exist $R^{\prime}>0$ and $k_{0} \in \mathbb{N}$ such that

$$
\rho\left(P_{k}\right) \cap\left(R-R^{\prime}, R+R^{\prime}\right)=\emptyset
$$

for all $k \geq k_{0}$. Take $0<\varepsilon<\varepsilon_{0}$ sufficiently small so that

$$
32 R \varepsilon<R^{\prime},
$$

$$
C_{\mathscr{F}}^{\prime} R^{4} \sqrt{\varepsilon}+C_{\mathscr{F}}^{\prime \prime} R^{3} \sqrt{\varepsilon}<\bar{\varepsilon},
$$

$$
C_{D \mathscr{F}} R^{3} \sqrt{\varepsilon}<\bar{\varepsilon}
$$


for constants $\varepsilon_{0}, C_{\mathscr{F}}^{\prime}, C_{\mathscr{F}}^{\prime \prime}$ and $C_{D \mathscr{F}}$ of Proposition 4.2. Finally, by assumptions (1.19) we know that there exists a $K \geq k_{0}$ such that

$$
m_{A D M}\left(M_{k}^{\prime}\right)<R \varepsilon^{3} \text { and } \frac{m_{A D M}\left(M_{k}^{\prime}\right)}{\sigma\left(M_{k}\right)}<\frac{\varepsilon}{32}
$$

for all $k \geq K$. The hypotheses of Proposition 4.2 are now satisfied and as a result we obtain (4.123). This completes our proof.

\section{Appendix: Inverting a geometrostatic manifold}

Recall that the Riemannian Schwarzschild manifold of mass $m_{1}=m$ is a geometrostatic manifold with a single point $p_{1}=0$ and that this manifold has an isometry which interchanges the two ends:

(A.1)

$F: \mathbb{R}^{3} \backslash\{0\} \rightarrow \mathbb{R}^{3} \backslash\{0\}$ defined by $F(y)=\left(\frac{m}{2}\right)^{2} \frac{y}{|y|^{2}}$ with $F^{-1}(x)=\left(\frac{m}{2}\right)^{2} \frac{x}{|x|^{2}}$.

That is the pullback metric of $g_{\mathrm{Sch}}$ as in 1.8 is

$$
\begin{aligned}
\left(F^{*} g_{\text {Sch }}\right)_{y} & =\left(1+\frac{m}{2|F(y)|}\right)^{4}\left(F^{*} \delta\right)_{y}=\left(1+\frac{2|y|}{m}\right)^{4}\left(\frac{m}{2}\right)^{4} \frac{1}{|y|^{4}} \delta_{y} \\
& =\left(\left(\frac{m}{2}\right) \frac{1}{|y|}+1\right)^{4} \delta_{y}=\left(g_{\text {Sch }}\right)_{y} .
\end{aligned}
$$

Similarly, if we apply an inversion to any geometrostatic manifold taking the end at infinity to an end at the origin and taking one of the other ends to the end at infinity we obtain an isometric geometrostatic manifold:

Theorem A.1. Let $\left(X, g_{X}\right)$ be the geometrostatic manifold:

(A.4) $X=\mathbb{R}^{n} \backslash\left\{x_{1}, \ldots, x_{n}\right\}$ with $g_{X}=\left(1+\sum_{i=1}^{n} \frac{\alpha_{X, i}}{\left|x-x_{i}\right|}\right)^{2}\left(1+\sum_{i=1}^{n} \frac{\beta_{X, i}}{\left|x-x_{i}\right|}\right)^{2} \delta_{x}$

Let

$$
F(y)=\alpha_{n} \beta_{n} \frac{y}{|y|^{2}}+x_{n} \text { so that } F^{-1}(x)=\alpha_{n} \beta_{n} \frac{x-x_{n}}{\left|x-x_{n}\right|^{2}} .
$$

Let $y_{0}=0$ and

$$
y_{j}=F^{-1}\left(x_{j}\right)=\alpha_{n} \beta_{n} \frac{x_{j}-x_{n}}{\left|x_{j}-x_{n}\right|^{2}}=\alpha_{n} \beta_{n} \frac{x_{j}-x_{n}}{r_{j n}^{2}} \quad \text { for } j=1, . ., n-1 .
$$

Let $\left(Y, g_{Y}\right)$ be the geometrostatic manifold

$$
Y=\mathbb{R}^{n} \backslash\left\{y_{0}, \ldots, y_{n-1}\right\} \text { with } g_{Y}=\left(1+\sum_{i=0}^{n-1} \frac{\alpha_{Y, i}}{\left|y-y_{i}\right|}\right)^{2}\left(1+\sum_{i=0}^{n-1} \frac{\beta_{Y, i}}{\left|y-y_{i}\right|}\right)^{2} \delta_{y}
$$


where

(A.8)

$$
\begin{aligned}
& \alpha_{Y, 0}=\alpha_{n} \\
& \beta_{Y, 0}=\beta_{n}
\end{aligned}
$$

$$
\begin{aligned}
& \alpha_{Y, j}=\frac{\beta_{X, j} \alpha_{n}}{r_{j, n}}=\frac{\beta_{X, j}\left|y_{j}\right|}{\beta_{n}} \\
& \beta_{Y, j}=\frac{\alpha_{X, j} \beta_{n}}{r_{j, n}}=\frac{\alpha_{X, j}\left|y_{j}\right|}{\alpha_{n}}
\end{aligned}
$$

Then $F: Y \rightarrow X$ is an isometry which maps the end at infinity for $Y$ to the end at $x_{n}$ for $X$ and the end at 0 for $Y$ to the end at infinity for $X$, and the end at $y_{j}$ for $Y$ to the end at $x_{j}$ for $X$.

The proof of this theorem follows a method of Misner in [Mis63]. We include it for completeness of exposition.

Proof. We need only prove $g_{Y}=F^{*} g_{X}$.

First observe that

$$
\left(F^{*} \delta\right)_{y}=\left(\alpha_{n} \beta_{n}\right)^{2} \frac{1}{|y|^{4}} \delta_{y} .
$$

So

$$
\begin{aligned}
\left(F^{*} g_{X}\right)_{y} & =\left(1+\sum_{j=1}^{n} \frac{\alpha_{X, j}}{\left|F(y)-x_{j}\right|}\right)^{2}\left(1+\sum_{j=1}^{n} \frac{\beta_{X, j}}{\left|F(y)-x_{j}\right|}\right)^{2}\left(F^{*} \delta\right)_{y} \\
& =\left(\left(\frac{\beta_{n}}{|y|}\right)\left(1+\sum_{j=1}^{n} \frac{\alpha_{X, j}}{\left|F(y)-x_{j}\right|}\right)\right)^{2}\left(\left(\frac{\alpha_{n}}{|y|}\right)\left(1+\sum_{j=1}^{n} \frac{\beta_{X, j}}{\left|F(y)-x_{j}\right|}\right)\right)^{2} \delta_{y} .
\end{aligned}
$$

We need only prove

$$
\begin{aligned}
& \left(\frac{\beta_{n}}{|y|}\right)\left(1+\sum_{j=1}^{n} \frac{\alpha_{X, j}}{\left|F(y)-x_{j}\right|}\right)=\left(1+\sum_{j=0}^{n-1} \frac{\beta_{Y, j}}{\left|y-y_{j}\right|}\right) \\
& \left(\frac{\alpha_{n}}{|y|}\right)\left(1+\sum_{j=1}^{n} \frac{\beta_{X, j}}{\left|F(y)-x_{j}\right|}\right)=\left(1+\sum_{j=0}^{n-1} \frac{\alpha_{Y, j}}{\left|y-y_{j}\right|}\right) .
\end{aligned}
$$

First observe that
(A.15) $\frac{\beta_{n}}{|y|}=\frac{\beta_{n}}{\left|y-y_{0}\right|}=\frac{\beta_{Y, 0}}{\left|y-y_{0}\right|}$ by the choice of $y_{0}=0$ and $\beta_{Y, 0}=\beta_{n}$,
(A.16) $\frac{\alpha_{n}}{|y|}=\frac{\alpha_{n}}{\left|y-y_{0}\right|}=\frac{\alpha_{Y, 0}}{\left|y-y_{0}\right|}$ by the choice of $y_{0}=0$ and $\alpha_{Y, 0}=\alpha_{n}$. 
Since $\alpha_{X, n}=\alpha_{n}$ and $\beta_{X, n}=\beta_{n}$ we have,
(A.17) $\frac{\beta_{n}}{|y|}\left(\frac{\alpha_{X, n}}{\left|F(y)-x_{n}\right|}\right)=\frac{\beta_{n}}{|y|}\left(\frac{\alpha_{n}}{\left.\left|\alpha_{n} \beta_{n} y /\right| y\right|^{2}+x_{n}-x_{n} \mid}\right)=\frac{\beta_{n} \alpha_{n}}{\left|\alpha_{n} \beta_{n} y /\right| y||}=1$,
(A.18) $\frac{\alpha_{n}}{|y|}\left(\frac{\beta_{X, n}}{\left|F(y)-x_{n}\right|}\right)=\frac{\alpha_{n}}{|y|}\left(\frac{\beta_{n}}{\left.\left|\alpha_{n} \beta_{n} y /\right| y\right|^{2}+x_{n}-x_{n} \mid}\right)=\frac{\alpha_{n} \beta_{n}}{\left|\alpha_{n} \beta_{n} y /\right| y||}=1$.

So we need only prove for $j=1, \ldots, n-1$ we have

$$
\begin{aligned}
& \left(\frac{\beta_{n}}{|y|}\right)\left(\frac{\alpha_{X, j}}{\left|F(y)-x_{j}\right|}\right)=\left(\frac{\beta_{Y, j}}{\left|y-y_{j}\right|}\right) \\
& \left(\frac{\alpha_{n}}{|y|}\right)\left(\frac{\beta_{X, j}}{\left|F(y)-x_{j}\right|}\right)=\left(\frac{\alpha_{Y, j}}{\left|y-y_{j}\right|}\right) .
\end{aligned}
$$

Now

$$
\begin{array}{ll}
\text { (A.21) } \quad\left|F(y)-x_{j}\right|^{2} & =\left|F(y)-F\left(y_{j}\right)\right|^{2} \\
\text { (A.22) } & =\left|\alpha_{n} \beta_{n} \frac{y}{|y|^{2}}+x_{n}-\alpha_{n} \beta_{n} \frac{y_{j}}{\left|y_{j}\right|^{2}}-x_{n}\right|^{2} \\
\text { (A.23) } & =\left(\alpha_{n} \beta_{n}\right)^{2}\left|\frac{y}{|y|^{2}}-\frac{y_{j}}{\left|y_{j}\right|^{2}}\right|^{2} \\
\text { (A.24) } & =\left.\frac{\left(\alpha_{n} \beta_{n}\right)^{2}}{|y|^{4}\left|y_{j}\right|^{4}}|| y_{j}\right|^{2} y-\left.|y|^{2} y_{j}\right|^{2} \\
\text { (A.25) } & =\frac{\left(\alpha_{n} \beta_{n}\right)^{2}}{|y|^{4}\left|y_{j}\right|^{4}}\left(\left|y_{j}\right|^{4}|y|^{2}+|y|^{4}\left|y_{j}\right|^{2}-2\left|y_{j}\right|^{2}|y|^{2} y \cdot y_{j}\right) \\
\text { (A.26) } & =\frac{\left(\alpha_{n} \beta_{n}\right)^{2}}{|y|^{2}\left|y_{j}\right|^{2}}\left(\left|y_{j}\right|^{2}+|y|^{2}-2 y \cdot y_{j}\right) \\
\text { (A.27) } & =\frac{\left(\alpha_{n} \beta_{n}\right)^{2}}{|y|^{2}\left|y_{j}\right|^{2}}\left|y-y_{j}\right|^{2} .
\end{array}
$$

Applying this to $y=y_{k}$ we have

$$
r_{k, j}^{2}=\left|x_{k}-x_{j}\right|^{2}=\frac{\left(\alpha_{n} \beta_{n}\right)^{2}}{\left|y_{k}\right|^{2}\left|y_{j}\right|^{2}}\left|y_{k}-y_{j}\right|^{2} .
$$

Applying it more generally for $j \neq n$ we have

$$
\begin{aligned}
& \text { (A.29) } \quad\left(\frac{\beta_{n}}{|y|}\right)\left(\frac{\alpha_{X, j}}{\left|F(y)-x_{j}\right|}\right)=\left(\frac{\beta_{n}}{|y|}\right)\left(\frac{\alpha_{X, j}|y|\left|y_{j}\right|}{\alpha_{n} \beta_{n}\left|y-y_{j}\right|}\right)=\frac{\beta_{Y, j}}{\left|y-y_{j}\right|} \\
& \text { (A.30) } \quad\left(\frac{\alpha_{n}}{|y|}\right)\left(\frac{\beta_{X, j}}{\left|F(y)-x_{j}\right|}\right)=\left(\frac{\alpha_{n}}{|y|}\right)\left(\frac{\beta_{X, j}|y|\left|y_{j}\right|}{\alpha_{n} \beta_{n}\left|y-y_{j}\right|}\right)=\frac{\alpha_{Y, j}}{\left|y-y_{j}\right|} .
\end{aligned}
$$


Corollary A.2. Since $F$ is an isometry which maps the end at infinity for $Y$ to the end at $x_{n}$ for $X$ these ends have the same ADM mass:

$$
m_{A D M}(Y)=m_{X, n} .
$$

Since $F$ is an isometry which maps the end at $y_{0}$ for $Y$ to the end at $\infty$ for $X$, these ends have the same ADM mass:

$$
m_{Y, 0}=m_{A D M}(X) .
$$

Proof. We also prove these equalities using (1.4) and (1.5) thus rederiving these equations that were stated without proof in Brill-Lindquist. We have by (1.4) that the ADM mass of the end at infinity for $Y$ satisfies:

$$
\begin{aligned}
& \left(\mathrm{A} .33 \mathrm{~m}_{A D M}(Y)=\sum_{j=0}^{n-1}\left(\alpha_{Y, j}+\beta_{Y, j}\right)=\alpha_{X, n}+\beta_{X, n}+\sum_{j=1}^{n-1}\left(\frac{\beta_{X, j} \alpha_{n}}{r_{j, n}}+\frac{\alpha_{X, j} \beta_{n}}{r_{j, n}}\right)\right. \\
& =\alpha_{X, n}+\beta_{X, n}+\sum_{j=1}^{n-1}\left(\frac{\beta_{X, j} \alpha_{X, n}+\alpha_{X, j} \beta_{X, n}}{r_{j, n}}\right)=m_{X, n} \text { by (1.5) }
\end{aligned}
$$

where $m_{X, n}$ is the ADM mass of the end at $x_{n}$ for $X$. This is also a consequence of the fact that $F$ is an isometry which maps the end at infinity for $Y$ to the end at $x_{n}$ for $X$. We have by (1.5) that the ADM mass of the end at $y_{0}$ for $Y$ satisfies

$$
\begin{aligned}
m_{Y, 0} & =\alpha_{Y, 0}+\beta_{Y, 0}+\sum_{j=1}^{n-1} \frac{\left(\beta_{Y, 0} \alpha_{Y, j}+\alpha_{Y, 0} \beta_{Y, j}\right)}{\left|y_{0}-y_{j}\right|} \\
& =\alpha_{n}+\beta_{n}+\sum_{j=1}^{n-1} \frac{\beta_{n}\left(\beta_{X, j} \alpha_{n} / r_{j, n}\right)+\alpha_{n}\left(\alpha_{X, j} \beta_{n} / r_{j, n}\right)}{\left(\alpha_{n} \beta_{n} / r_{j, n}\right)} \\
& =\alpha_{X, n}+\beta_{X, n}+\sum_{j=1}^{n-1}\left(\beta_{X, j}+\alpha_{X, j}\right)=m_{A D M}(X) \text { by (1.4) }
\end{aligned}
$$

where $m_{A D M}(X)$ is the ADM mass of the end at infinity for $X$. This is also a consequence of the fact that $F$ is an isometry which maps the end at $y_{0}$ for $Y$ to the end at $\infty$ for $X$. Recall that by (A.28) we have

$$
\left|y_{k}-y_{j}\right|=\frac{r_{k, j}}{\left(\alpha_{n} \beta_{n}\right)}\left|y_{k}\right|\left|y_{j}\right|=\frac{r_{k, j}}{\left(\alpha_{n} \beta_{n}\right)}\left(\frac{\alpha_{n} \beta_{n}}{r_{k, n}}\right)\left(\frac{\alpha_{n} \beta_{n}}{r_{j, n}}\right)=\frac{\alpha_{n} \beta_{n} r_{k, j}}{r_{k, n} r_{j, n}}
$$


We can combine this with (1.5) to show that the ADM mass of the end at $y_{k} \neq y_{0}$ for $Y$ satisfies

$$
\begin{aligned}
m_{Y, k}= & \alpha_{Y, k}+\beta_{Y, k}+\frac{\left(\beta_{Y, k} \alpha_{Y, 0}+\beta_{Y, 0} \alpha_{Y, k}\right)}{\left|y_{k}-0\right|}+\sum_{j \neq k, j=1}^{n-1} \frac{\left(\beta_{Y, k} \alpha_{Y, j}+\beta_{Y, j} \alpha_{Y, k}\right)}{\left|y_{k}-y_{j}\right|} \\
= & \frac{\beta_{X, k} \alpha_{n}}{r_{k, n}}+\frac{\alpha_{X, k} \beta_{n}}{r_{k, n}}+\left(\frac{\alpha_{X, k} \beta_{n} \alpha_{n}}{r_{k, n}}+\frac{\beta_{n} \beta_{X, k} \alpha_{n}}{r_{k, n}}\right) \frac{r_{k, n}}{\alpha_{n} \beta_{n}} \\
& +\sum_{j \neq k, j=1}^{n-1} \frac{\left(\alpha_{X, k} \beta_{n} / r_{k, n}\right)\left(\beta_{X, j} \alpha_{n} / r_{j, n}\right)+\left(\alpha_{X, j} \beta_{n} / r_{j, n}\right)\left(\beta_{X, k} \alpha_{n} / r_{k, n}\right)}{\left(\alpha_{n} \beta_{n} r_{k, j}\right) /\left(r_{k, n} r_{j, n}\right)} \\
= & \frac{\beta_{X, k} \alpha_{X, n}+\alpha_{X, k} \beta_{X, n}}{\left|x_{k}-x_{n}\right|}+\left(\alpha_{X, k}+\beta_{X, k}\right)+\sum_{j \neq k, j=1}^{n-1} \frac{\left(\alpha_{X, k} \beta_{X, j}+\alpha_{X, j} \beta_{X, k}\right)}{r_{k, j}} \\
= & \left(\alpha_{X, k}+\beta_{X, k}\right)+\sum_{j \neq k, j=1}^{n} \frac{\left(\alpha_{X, k} \beta_{X, j}+\alpha_{X, j} \beta_{X, k}\right)}{r_{k, j}}=m_{X, k} \text { by 1.5 }
\end{aligned}
$$

where $m_{X, k}$ is the ADM mass of the end at $x_{k} \neq x_{n}$ for $X$. This is also a consequence of the fact that $F$ maps the end of $Y$ at $y_{k}$ to the end of $X$ at $x_{k}$ for $k=1, \ldots, n-1$.

\section{Acknowledgment.}

The authors would like to thank Mingliang Cai, Qing Chen, Xiuxiong Chen, Piotr Chrusciel, Greg Galloway, Sen Hu and Jim Isenberg for organizing the July 2014 Geometric Analysis and Relativity Conference at the University of Science and Technology of China at which this collaboration commenced. We would also like to thank Greg Galloway, David Maxwell, Richard Schoen, and Daniel Pollack for organizing the Banff Geometric Analysis and General Relativity Workshop in July 2016 during which the final version of this work was formulated.

Prof. Sormani's research is funded in part by a PSC CUNY Research Grant and by NSF DMS 1309360. Prof. Stavrov's research is funded in part by travel grants from Lewis \& Clark College.

\section{Bibliography}

[BL63] Dieter Brill and Richard Lindquist. Interaction Energy in Geometrostatics. Article Physical Review, 131:471-476, 1963.

[Bra01] Hubert L. Bray. Proof of the Riemannian Penrose inequality using the positive mass theorem. J. Differential Geom., 59(2):177-267, 2001.

[CGT82] Jeff Cheeger, Mikhail Gromov, and Michael Taylor. Finite propagation speed, kernel estimates for functions of the Laplace operator, and the geometry of complete Riemannian manifolds. J. Differential Geom., 17(1):15-53, 1982.

[CM11] Tobias Holck Colding and William P. Minicozzi, II. A course in minimal surfaces, volume 121 of Graduate Studies in Mathematics. American Mathematical Society, Providence, RI, 2011.

[HI01] Gerhard Huisken and Tom Ilmanen. The inverse mean curvature flow and the Riemannian Penrose inequality. J. Differential Geom., 59(3):353-437, 2001. 
[HLS16] Lan-Hsuan Huang, Dan A Lee, and Christina Sormani. Intrinsic flat stability of the positive mass theorem for graphical hypersurfaces of Euclidean space. Journal fur die Riene und Angewandte Mathematik (Crelle's Journal), pages 1-31, 2016.

[Jau14] J Jauregui. On the lower semicontinuity of the adm mass. Comm. Anal. Geom., 26, (1), 2018.

[LS13] Sajjad Lakzian and Christina Sormani. Smooth convergence away from singular sets. Comm. Anal. Geom., 21(1):39-104, 2013.

[L09] Dan A. Lee. On the near-equality case of the positive mass theorem. Duke., 148(1):63-80, 2009.

[LS14] Dan A. Lee and Christina Sormani. Stability of the positive mass theorem for rotationally symmetric Riemannian manifolds. J. Reine Angew. Math., 686:187-220, 2014.

[LS15] Philippe G. LeFloch and Christina Sormani. The nonlinear stability of rotationally symmetric spaces with low regularity. J. Funct. Anal., 268(7):2005-2065, 2015.

[L44] A A Lichnerowicz. Sur l'intgration des quations d'Einstein. J. Math. Pures Appl., 23:26-63, 1944.

[Mis63] Charles W. Misner. The method of images in geometrostatics. Ann. Physics, 24:102-117, 1963.

[SW11] Christina Sormani and Stefan Wenger. The intrinsic flat distance between Riemannian manifolds and other integral current spaces. J. Differential Geom., 87(1):117-199, 2011.

[SY79] Richard Schoen and Shing Tung Yau. On the proof of the positive mass conjecture in general relativity. Comm. Math. Phys., 65(1):45-76, 1979. 